J. DIFFERENTIAL GEOMETRY

66 (2004) 303-343

\title{
HITCHIN'S CONNECTION AND DIFFERENTIAL OPERATORS WITH VALUES IN THE DETERMINANT BUNDLE
}

\author{
Xiaotao Sun \& I-Hsun Tsai
}

\begin{abstract}
For a family of smooth curves, we have the associated family of moduli spaces of stable bundles with fixed determinant on the curves. There exists a so-called theta line bundle on the family of moduli spaces. When the Kodaira-Spencer map of the family of curves is an isomorphism, we prove in this paper an identification theorem between sheaves of differential operators on the theta line bundle and higher direct images of vector bundles on curves. As an application, the so-called Hitchin connection on the direct image of (powers of) the theta line bundle is derived naturally from the identification theorem. A logarithmic extension to certain singular stable curves is also presented in this paper.
\end{abstract}

\section{Introduction}

Let $\mathcal{M}$ be a smooth variety over any algebraically closed field of characteristic zero and $S \rightarrow \mathcal{M}$ be a smooth, flat morphism. For a smooth family $\pi: X \rightarrow S$ of curves and a vector bundle $E$ on $X$, A. Beilinson and V. Schechtman defined in [2] (see also [4]) the so-called trace complexes ${ }^{\operatorname{tr}} \mathcal{A}_{E / \mathcal{M}}^{\bullet},{ }^{\operatorname{tr}} \mathcal{A}_{E}^{\bullet}$ on $X$, together with algebra structures on them, such that $R^{0} \pi_{*}\left({ }^{\operatorname{tr}} \mathcal{A}_{E / \mathcal{M}}^{\bullet}\right), R^{0} \pi_{*}\left({ }^{\operatorname{tr}} \mathcal{A}_{E}^{\bullet}\right)$ are canonically isomorphic to the Atiyah algebras $\mathcal{A}_{\lambda_{E} / \mathcal{M}}, \mathcal{A}_{\lambda_{E}}$ of the determinant bundle $\lambda_{E}=\operatorname{det} R \pi_{*} E$ of the family (see Proposition 3.3 for details), where $\mathcal{A}_{\lambda_{E}}$, as a sheaf on $S$, is just $\mathcal{D} \leq 1\left(\lambda_{E}\right)$, the sheaf of differential operators of order $\leq 1$ with values in $\lambda_{E}$, and similarly $\mathcal{A}_{\lambda_{E} / \mathcal{M}} \subset \mathcal{A}_{\lambda_{E}}$ corresponds to relative differential operators. It is generalized by Y.-L. Tong and the

Sun is supported by the outstanding young grant of NFSC at number 10025103 and a grant of RGC of Hong Kong at RGC code 7131/02P. Tsai has been supported in part by a grant from National Science Council of Taiwan.

Received 11/18/2003. 
second author $([\mathbf{1 9}])$ to the families $\pi: \widetilde{X} \rightarrow \widetilde{S}$ of stable curves. In this paper, we prove firstly, under the assumption that Kodaira-Spencer maps of the families are isomorphisms, one can canonically produce locally free sheaves $\mathcal{G}_{E}, S\left(\mathcal{G}_{E}\right)$ on $X$ (resp. on $\widetilde{X}$ ) instead of the above trace complexes such that one has the following identification theorem:

\section{Theorem 1.1.}

i) If $E$ is fiberwise stable and the Kodaira-Spencer map

$$
\mathrm{KS}: T_{S / \mathcal{M}} \rightarrow R^{1} \pi_{*}(\mathbb{E})
$$

is an isomorphism, where $\mathbb{E}:=\mathcal{E} n d^{0}(E)$ is the trace free part of $\mathcal{E} n d(E)$, then there exists a canonical isomorphism

$$
\phi: R^{1} \pi_{*}\left(\mathcal{G}_{E}\right) \cong \mathcal{D} \leq 1 / \mathcal{M}\left(\lambda_{\mathbb{E}}\right) .
$$

ii) If $\mathrm{KS}_{S}: T_{S} \rightarrow R^{1} \pi_{*} \mathcal{A}_{E / S}^{0}$ is an isomorphism, then (after shrinking $\mathcal{M}$ enough) $\phi$ extends to an isomorphism (cf. Theorem 2.5 for details)

$$
\phi=\phi_{-\lambda}: R^{1} \pi_{*} S\left(\mathcal{G}_{E}\right) \cong \mathcal{D}_{S}^{\leq 1}\left(\lambda_{\mathbb{E}}\right) .
$$

The most technical part of this paper is devoted to proof of the $i d e n$ tification theorem and its generalization to stable curves $\pi: \widetilde{X} \rightarrow \widetilde{S}$ (cf. Theorem 2.13). There is an interesting example of the families $S \rightarrow \mathcal{M}$, $\pi: X \rightarrow S$ which satisfy the assumptions in our theorem. Let $\mathcal{C} \rightarrow \mathcal{M}$ be a family of smooth curves of genus $g \geq 2$ and $f: S \rightarrow \mathcal{M}$ be the associated family of moduli spaces of stable bundles of rank $r \geq 2$ with a fixed determinant. Let $\pi: X=\mathcal{C} \times{ }_{\mathcal{M}} S \rightarrow S, E$ a universal bundle on $X$. Then KS: $T_{S / \mathcal{M}} \rightarrow R^{1} \pi_{*}(\mathbb{E})$ is an isomorphism. If furthermore, for any $b \in \mathcal{M}, T_{\mathcal{M}, b} \cong H^{1}\left(\mathcal{C}_{b}, T_{\mathcal{C}_{b}}\right)$, then $\mathrm{KS}_{S}: T_{S} \rightarrow R^{1} \pi_{*} \mathcal{A}_{E / S}^{0}$ is an isomorphism (cf. Propsition 4.5). A family $\mathcal{C} \rightarrow \mathcal{M}$ as such (called good family for convenience) does exist in algebraic category of characteristic zero (in fact, $\mathcal{M}$ is a moduli space of curves with level structures, which is a finite Galois cover of the moduli space $\mathcal{M}_{g}$ of curves). We will fix such a family in (the most part of) this paper. Let $\Theta$ be the theta line bundle on $S$ and, for any $k$, write $\Theta^{k}=\lambda_{\mathbb{E}}^{\mu}$ for some $\mu \in \mathbb{Q}$. Then our identification theorem gives immediately a new construction of Hitchin's connection on $\mathcal{V}_{k}=f_{*}\left(\Theta^{k}\right)$.

Corollary 1.2. There exists a unique projective heat operator,

$$
\mathrm{H}: T_{\mathcal{M}} \rightarrow f_{*} \mathcal{D}_{\bar{S}}^{\leq 2}\left(\Theta^{k}\right) / \mathcal{O}_{\mathcal{M}}
$$

which lifts Hitchin's symbol map $f_{*} \delta_{\mathrm{H}}: T_{\mathcal{M}} \rightarrow f_{*} S^{2} T_{S / \mathcal{M}}$. 
The construction of the above heat operator is purely algebraic by playing diagram game. The map $\delta_{\mathrm{H}}: f^{*} T_{\mathcal{M}} \rightarrow S^{2} T_{S / \mathcal{M}}$ is a connecting map in a long exact sequence of cohomologies, which coincides with Hitchin's symbol map over complex number field. The reason we restrict ourselves in characteristic zero is: the theory of Beilinson-Schechtman (thus our identification theorem) is in characteristic zero. On the other hand, Tsuchiya-Ueno-Yamada [20] construct a conformal block space associated to each pointed-stable curve, these spaces form a vector bundle on the moduli space of stable curves. They also construct a logarithmic projective connection (WZW connection) on this vector bundle of conformal blocks. It is proved that the algebro-geometric analogues of conformal block spaces for smooth curves are the spaces of global sections of $\Theta^{k}$. Y. Laszlo [13] proves that on the moduli space $\mathcal{M}_{g, 1}$ of smooth curves with a point Hitchin's connection coincides with the WZW connection (note that $\mathcal{M}_{g, 1}$ is of dimension larger than our $\mathcal{M}$ ). However, for singular stable curves, the algebro-geometric analogues of conformal block spaces are still unknown (by our knowledge). One reason, among others, is simply that degeneration of moduli spaces of stable $S L(r)$-bundles is still an open problem (cf. [6], [7] for comments on this problem). Thus it seems to us that a geometrically meaningful extension of $\mathcal{V}_{k}=f_{*}\left(\Theta^{k}\right)$ to the boundary with logarithmic connection would be interesting. Indeed, the question of studying the behaviour of spaces of generalized theta functions under degeneration of the curves was already mentioned in [10], p. 350, and the question of studying the (logarithmic) extension of the connection was asked in an unpublished manuscript of Beilinson and Kazhdan [1], p. 27, where they produced the connection for the case of smooth curves.

Let $\widetilde{S}, \widetilde{\mathcal{M}}$ be smooth varieties and $B=\widetilde{\mathcal{M}} \backslash \mathcal{M}$ be a normal crossing divisor. Let $\widetilde{f}: \widetilde{S} \rightarrow \widetilde{\mathcal{M}}$ be a smooth flat morphism, $\pi: \widetilde{X} \rightarrow \widetilde{S}$ be a family of stable curves and $E$ a fiberwise stable vector bundle on $\widetilde{X}$. Let $W=\widetilde{f}^{-1}(B) \subset \widetilde{S}$. Then the logarithmic identification theorem can be proved similarly as in the smooth case.

\section{Theorem 1.3.}

i) If $\mathrm{KS}: T_{\widetilde{S} / \widetilde{\mathcal{M}}} \rightarrow R^{1} \pi_{*}(\mathbb{E})$ is an isomorphism, where $\mathbb{E}:=\mathcal{E} n d^{0}(E)$, then there exists a canonical isomorphism

$$
\phi: R^{1} \pi_{*}\left(\mathcal{G}_{E}\right) \cong \mathcal{D} \underset{\widetilde{S} / \widetilde{\mathcal{M}}}{\leq 1}\left(\lambda_{\mathbb{E}}\right)
$$


ii) If $\mathrm{KS}_{\widetilde{S}}: T_{\widetilde{S}}(\log W) \rightarrow R^{1} \pi_{*} \mathcal{A}_{E / \widetilde{S}}^{0}(\log D)$ is an isomorphism, then (after shrinking $\widetilde{\mathcal{M}}$ enough) $\phi$ extends to an isomorphism (cf. Theorem 2.13 for details)

$$
\phi=\phi_{-\lambda}: R^{1} \pi_{*} S\left(\mathcal{G}_{E}\right) \cong \mathcal{D}_{\widetilde{S}}^{\leq 1}\left(\lambda_{\mathbb{E}}\right)(\log W) .
$$

If $\widetilde{\mathcal{C}} \rightarrow \widetilde{\mathcal{M}}$ is a family of stable curves, let $\mathcal{U}(r, d) \rightarrow \widetilde{\mathcal{M}}$ be the family of moduli spaces of semistable (for canonical polarization) torsion-free sheaves of rank $r$ and degree $d$. Fix a line bundle $\mathcal{N}$ on $\widetilde{\mathcal{C}}$ of relative degree $d$, let $f: S \rightarrow \mathcal{M}$ be the family of moduli spaces of stable bundles of rank $r$ with fixed determinant $\left.\mathcal{N}\right|_{C}([C] \in \mathcal{M})$. Let $Z \subset \mathcal{U}(r, d)$ be the Zariski closure of $S$ in $\mathcal{U}(r, d)$ and $f_{Z}: Z \rightarrow \widetilde{\mathcal{M}}$ the induced morphism. Let $\widetilde{f}: \widetilde{S} \subset Z \rightarrow \widetilde{\mathcal{M}}$ be the open set of $Z$ consisting of stable bundles. Let $\pi: \widetilde{X}=\widetilde{\mathcal{C}} \times \mathcal{M} \widetilde{S} \rightarrow \widetilde{S}$ and $E$ the universal bundle on $\widetilde{X}$. Note that for general reducible stable curves $\widetilde{\mathcal{C}_{b}}(b \in B)$, the (open) moduli spaces $\widetilde{f}^{-1}(b)$ hasn't been well understood yet, and in particular we do not know if the Kodaira-Spencer map in Theorem $1.3 \mathrm{i}$ ) is an isomorphism. On the other hand it is better understood for those irreducible stable curves and reducible stable curves whose dual diagrams are trees. Thus we will assume in this paper that the singular fibers of $\widetilde{\mathcal{C}} \rightarrow \widetilde{\mathcal{M}}$ are (irreducible or reducible) stable curves with only one node. Then $\tilde{f}$ : $\widetilde{S} \rightarrow \widetilde{\mathcal{M}}$ is smooth and the Kodaira-Spencer map in Theorem $1.3 \mathrm{i}$ ) is an isomorphism (cf. Lemma 4.4). Let $\widetilde{\mathcal{M}}_{g}$ be the moduli space of stable curves with level structures as in Looijenga's paper [14], with the universal family $\Pi: \widetilde{\mathcal{C}} \rightarrow \widetilde{\mathcal{M}}_{g}$. On a neighbourhood of $m \in \widetilde{\mathcal{M}}_{g}$, the family is the versal deformation of $\widetilde{\mathcal{C}}_{m}$. In particular, KS: $T_{\widetilde{\mathcal{M}}}(B) \rightarrow$ $R^{1} \Pi_{*} \omega_{\widetilde{\mathcal{C}} / \widetilde{\mathcal{M}}}^{-1}$ is an isomorphism, which implies that the Kodaira-Spencer map in Theorem 1.3 ii) is an isomorphism (cf. Proposition 4.5). We will fix $\widetilde{\mathcal{M}} \subset \widetilde{\mathcal{M}}_{g}$ consisting of stable curves with at most one double point and also fix the family $\widetilde{\mathcal{C}} \rightarrow \widetilde{\mathcal{M}}$ in this paper. It is known that $B=\widetilde{\mathcal{M}} \backslash \mathcal{M}$ is a normal crossing divisor (cf. [14]).

Corollary 1.4. There exists a unique projective heat operator

$$
\widetilde{\mathrm{H}}: T_{\widetilde{\mathcal{M}}}(B) \rightarrow \widetilde{f}_{*} \mathcal{D}_{\widetilde{S}}^{\leq 2}\left(\Theta^{k}\right) / \mathcal{O}_{\widetilde{\mathcal{M}}}
$$

that lifts Hitchin's symbol map (in the extended sense)

$$
\widetilde{f}_{*} \delta_{\mathrm{H}}: T_{\widetilde{\mathcal{M}}}(B) \rightarrow \widetilde{f}_{*} S^{2} T_{\widetilde{S} / \widetilde{\mathcal{M}}} .
$$


Moreover, $\widetilde{f}_{*}\left(\Theta^{k}\right)$ is a coherent sheaf on $\widetilde{\mathcal{M}}$.

After defining the extended Hitchin's symbol map, the proof goes through by using the logarithmic version of identification theorem. It is important that the vanishing of $R^{1} f_{*} \mathcal{O}_{S}$ is not needed in our proof both for smooth case and singular case (so that our method can be extended to some stable curves, where such a vanishing may not hold). The nontrivial part is the proof of coherence of $\widetilde{f}_{*}\left(\Theta^{k}\right)$ on $\mathcal{M}$ since $\widetilde{f}: \widetilde{S} \rightarrow \widetilde{\mathcal{M}}$ is not proper. To prove it, we prove (cf. Proposition 4.9) that the fibres of $\widetilde{f}: \widetilde{S} \rightarrow \widetilde{\mathcal{M}}$ is dense in the fibres of $f_{Z}: Z \rightarrow$ $\widetilde{\mathcal{M}}$, which partially confirms a conjecture of D. S. Nagaraj and C. S. Seshadri (cf. Conjecture (a) at page 136 of [15]). We believe that such a coherent extension remains true on $\widetilde{M}_{g}$ of all stable curves; this is not, however, proved in the present paper. On the other hand, if it can be proved that the compactification $Z$ is Cohen-Macaulay with vanishing $H^{1}\left(f_{Z}^{-1}(b), \Theta^{k}\right)(b \in B)$, then the coherent extension is a vector bundle whose fibers are spaces of generalized theta functions on certain moduli spaces. This seems to be true for rank two case.

Basically our approach works equally well both for the general case [10] $(g \geq 3)$ and special cases (e.g., $g=2$ and $r=2)$ [8], with the difference arising from the fact that in $g=r=2$ case our approach shall work with a Quot scheme $\mathcal{R}^{s s}$ which has a good quotient $\mathcal{R}^{s s} \rightarrow S$ where $S$ is the family of moduli spaces of S-equivalence classes of semistable vector bundles. As this will introduce additional details, we leave the case $g=r=2$ to future discussions.

Hitchin remarked in [10], p. 350, "Many aspects of the connection have so far resisted attack by the methods presented here...". Besides the degeneration problem treated here, it seems that two major problems remain poorly understood:

i) the unitary structure of the connection,

ii) (local) monodromy representation of the connection,

although there are discussions on these problems in the literature. For ii) we refer to [8] for interesting results for $g=r=2$ case along the line of Hitchin's approach; for comments on more general cases, see [8], Subsection 4.5 and references therein. We content ourselves by remarking that the methods for studying the monodromy representation (of the mapping class group) have largely been confined to those of topology and conformal field theory. An algebro-geometric picture is still lacking for the understanding (note that [8] merely found local monodromy 
representation; "local" means at $\infty$ ). In the spirit of the work $[\mathbf{1 0}],[\mathbf{1 3}]$ and $[8]$, we feel it worthy of being presented, among other things, the present proof of the existence of the logarithmic Hitchin's connection (beyond the explicit $g=r=2$ case of [8]), as a further step towards algebro-geometric aspects of these development. In our opinion it seems plausible that some explicit information about the (local) monodromy representation could be available via our approach. Indeed in the first version of this paper we produced a more explicit formula of the connection, at the expense that a lot more technical computations would be performed, while the present approach simplified considerably via use of Proposition 2.3 which we later discovered to be useful (in fact we came to pay more attention to this proposition via discussions with Hélène Esnault when we finished the first version including the case $g=r=2$ ). For the sake of conceptual simplicity we decided to take the present approach, and leave the more technical treatment (including the case $g=r=2$ ) to future discussions. We remark that there has been a speculation about whether monodromy representations factor through a finite group; see [3] where this speculation was attributed to M. Kontsevich. We had also met with mathematicians who asked about (and/or suggested the study of) the characteristic $p>0$ situation, including the related issues such as the notion of $p$-curvature of the connection, in connection to the highly speculative relation between the above Kontsevich conjecture and some conjectures of Grothendieck (cf. [12]). Unfortunately at this time of writing we are not feeling competent enough to comment on any one of these issues, based on results of the present work. Perhaps some further studies along these directions might be interesting. Besides generalizing Beilinson-Schechtman theory (thus our construction) to characteristic $p>0$, the logarithmic extension suggests that residue of the connection at the boundary gives an operator on the (projective) space of generalized theta functions on certain moduli space, which should be (direct sum of) spaces of generalized theta functions on moduli spaces of parabolic bundles over a smooth curve of lower genus (normalization of the singular curve $\widetilde{\mathcal{C}_{b}}$ ). It would be interesting to understand the relationship between the residue operator and the above factorization of generalized theta functions.

Those who want to know some historical accounts of Hitchin's connection and its relation to other branches of mathematics, are referred to $[\mathbf{1 0}],[\mathbf{8}]$ and references therein. 
In Section 2, we construct Hitchin's connection and its logarithmic extension under assumption of Theorem 2.5, Lemma 2.4 and Theorem 2.13. It is enough to read this section for a reader who is only interested in Hitchin's connection. Section 3 is devoted to the proof of Theorem 2.5 and Lemma 2.4. In Section 4, we indicate the modifications of arguments in Section 3 to prove Theorem 2.13. Most of Section 4 is devoted to prove the coherence of $\widetilde{f}_{*}\left(\Theta^{k}\right)$ on $\widetilde{\mathcal{M}}$.

Remark that some ideas closely related to Section 3 of this paper were briefly discussed in Sections 9, 10 of [9] where the subject matter is considered for moduli space of (stable) $G$-bundles, with $G$ being complex semisimple, connected and simply-connected.

Acknowledgements. We would like to express our deep gratitude to Hélène Esnault for the great impact of her ideas on this work. Indeed countless discussions with her in an earlier stage of this work either directly allowed us to grasp several key ideas, or contributed in an essential way to shaping ourselves towards the present approach. This joint work was initiated while both of us were visiting Essen (though a few topics involved in this work had already been studied by three of us independently). We wish to thank heartily Hélène Esnault and Eckart Viehweg for their warm hospitality and the excellent working conditions of Essen, that made our visit and this work possible. The anonymous referees have given us a critical analysis of several related topics that would be worth further pursuit; their thorough reading of this paper and supply of some relevant information have led to substantial improvement on our presentation. We thank them very much.

\section{Heat operators and cohomology of sheaves}

As in the introduction, $\mathcal{M} \subset \widetilde{\mathcal{M}}_{g}$ denote the smooth moduli spaces of curves with level structures $([\mathbf{1 4}])$. Let $\widetilde{\mathcal{M}} \subset \widetilde{\mathcal{M}}_{g}$ be the open set consisting of stable curves with at most one double point (thus $\operatorname{codim}\left(\widetilde{\mathcal{M}}_{g} \backslash \widetilde{\mathcal{M}}\right) \geq 2$ ). We will fix the families of curves $\mathcal{C} \rightarrow \mathcal{M}$, $\widetilde{\mathcal{C}} \rightarrow \widetilde{\mathcal{M}}$ which satisfy the assumptions on Kodaira-Spencer maps. Fix a line bundle $\mathcal{N}$ of relative degree $d$ on $\widetilde{\mathcal{C}} \rightarrow \widetilde{\mathcal{M}}$, let $f: S \rightarrow \mathcal{M}$ be the family of moduli spaces of stable bundles of rank $r$ with fixed determinant $\mathcal{N}_{b}:=\left.\mathcal{N}\right|_{\mathcal{C}_{b}}(b \in \mathcal{M})$ on $\mathcal{C}_{b}$. Let $\pi: X=\mathcal{C} \times{ }_{\mathcal{M}} S \rightarrow S$ be the pullback of $\mathcal{C} \rightarrow \mathcal{M}$ via $f: S \rightarrow \mathcal{M}$.

Remark 2.1. If $(d, r)=1$, there exists a universal bundle $E$ on $X$. In general $S$ is a good quotient of a Hilbert quotient scheme, denoted 
by $\mathcal{R}^{s}$, such that there is a universal bundle $E$ on $X_{\mathcal{R}^{s}}:=\mathcal{C} \times \mathcal{M} \mathcal{R}^{s}$. $E$ may not descend to $X$, but objects such as $\mathcal{E} n d^{0}(E), \mathcal{G}_{E},{ }^{\text {tr }} \mathcal{A}_{E}^{\bullet}$ (and other relevant constructions that will be discussed later in this section) do descend. Recall that a sheaf $\mathcal{F}$ on $X_{\mathcal{R}^{s}}$ descends to $X$ if the action, of scalar automorphisms of $E$ (relative to $\mathcal{R}^{s}$ ), on $\mathcal{F}$ is trivial, e.g., [11].

Without the danger of confusion we will henceforth be working as if a universal bundle $E$ existed on $X$.

Let $\Theta$ be the theta line bundle on $S$. For a sheaf $\mathcal{F}$ on $X$,

$$
\lambda_{\mathcal{F}}=\bigotimes_{q \geq 0} \operatorname{det} R^{q} \pi_{*} \mathcal{F}^{(-1)^{q}}
$$

denotes the Knudsen-Mumford determinant bundle on $S$ (cf. [2]). As usual $T_{S}, K_{S}$ denote tangent bundle, canonical bundle; $T_{S / \mathcal{M}}, K_{S / \mathcal{M}}$ denote the relative counterparts. Assume that $K_{S / \mathcal{M}}=\Theta^{-\lambda}$, where $\lambda=2(r, d)$ is the same notation used by Hitchin (cf. (2.8) in [10]). Let $\mathcal{D}_{S}^{\leq i}\left(\Theta^{k}\right)$ be the sheaf of differential operators on $\Theta^{k}$ of order $\leq i$, and by $\epsilon$ the symbol map of differential operators. Let $\mathcal{W}\left(\Theta^{k}\right):=\mathcal{D}_{S}^{\leq 1}\left(\Theta^{k}\right)+$ $\mathcal{D}_{S / \mathcal{M}}^{\leq 2}\left(\Theta^{k}\right)($ cf. $[\mathbf{1 0}],[\mathbf{8}])$, one has an exact sequence

$$
0 \rightarrow \mathcal{D} \underset{S / \mathcal{M}}{\leq 2}\left(\Theta^{k}\right) \rightarrow \mathcal{W}\left(\Theta^{k}\right) \stackrel{\sigma}{\rightarrow} f^{*} T_{\mathcal{M}} \rightarrow 0 .
$$

Definition 2.2 (cf. [8], 2.3.2). A heat operator $\mathrm{H}$ on $\Theta^{k}$ is an $\mathcal{O}_{S^{-}}$ map $f^{*} T_{\mathcal{M}} \stackrel{\mathrm{H}}{\longrightarrow} \mathcal{W}\left(\Theta^{k}\right)$ which, while composed with $\sigma$ above, is the identity. A projective heat operator $\mathrm{H}: T_{\mathcal{M}} \rightarrow f_{*} \mathcal{W}\left(\Theta^{k}\right) / \mathcal{O}_{\mathcal{M}}$ is an $\mathcal{O}_{\mathcal{M}^{-}}$ map such that any local lifting is a heat operator. The symbol map of $\mathrm{H}$ is $\epsilon \circ \mathrm{H}: f^{*} T_{\mathcal{M}} \rightarrow S^{2} T_{S / \mathcal{M}}$. A heat operator $\mathrm{H}$ induces an $\mathcal{O}_{\mathcal{M}}$-map (denoted by the same $\mathrm{H}$ ) $\mathrm{H}: T_{\mathcal{M}} \rightarrow f_{*} \mathcal{W}\left(\Theta^{k}\right)$. The heat operator and the preceding induced map will be used interchangeably throughout. A (projective) heat operator on $\Theta^{k}$ determines a (projective) connection $\nabla^{\mathrm{H}}$ on $f_{*} \Theta^{k}$ in a natural way $[\mathbf{8}]$.

We recall firstly a general result (forget moduli) of G. Faltings (cf. Proposition IV.5 of [5]). Let $Z \rightarrow S$ be smooth and $\mathcal{K}=K_{Z / S}$. For any line bundle $\mathcal{L}$, consider

$$
\begin{gathered}
0 \rightarrow \mathcal{D}_{Z / S}^{\leq 1}(\mathcal{L}) \rightarrow \mathcal{D}_{Z / S}^{\leq 2}(\mathcal{L}) \stackrel{\epsilon_{2}}{\longrightarrow} S^{2} T_{Z / S} \rightarrow 0 \\
0 \rightarrow \mathcal{D} \frac{\leq 1}{Z / S}(\mathcal{L}) \rightarrow S^{2} \mathcal{D} \frac{\leq 1}{Z / S}(\mathcal{L}) \stackrel{S^{2} \epsilon_{1}}{\longrightarrow} S^{2} T_{Z / S} \rightarrow 0 .
\end{gathered}
$$


For any $\rho \in H^{0}\left(Z, S^{2} T_{Z / S}\right)$, let $a(\mathcal{L}, \rho), b(\mathcal{L}, \rho)$ be the obstruction classes to lift $\rho$ to $H^{0}\left(Z, \mathcal{D} \frac{\leq 2}{Z / S}(\mathcal{L})\right)$ and to $H^{0}\left(Z, S^{2} \mathcal{D} \frac{\leq 1}{Z / S}(\mathcal{L})\right)$ respectively. Then:

Proposition 2.3. Under $\mathcal{D}_{Z / S}^{\leq 1}\left(\mathcal{L}^{k}\right) \cong \mathcal{D}_{Z / S}^{\leq 1}(\mathcal{L})$, one has:

i) $b\left(\mathcal{L}^{k}, \rho\right)=k b(\mathcal{L}, \rho)$ for any $k \in \mathbb{Q}$.

ii) $a\left(\mathcal{O}_{Z}, \rho\right) \in H^{1}\left(\mathcal{O}_{Z}\right) \oplus H^{1}\left(T_{Z / S}\right)$ has zero projection in $H^{1}\left(\mathcal{O}_{Z}\right)$.

iii) There is a class $c(\mathcal{K}, \rho) \in H^{1}\left(\mathcal{O}_{Z}\right)$, independent of $\mathcal{L}$, such that

$$
2 a(\mathcal{L}, \rho)=b(\mathcal{L}, \rho)+{ }^{t} b\left(\mathcal{L}^{-1} \otimes \mathcal{K}, \rho\right)+c(\mathcal{K}, \rho) .
$$

We come back to construct the projective connection. Recall that

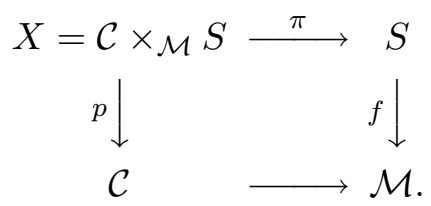

For simplicity, we assume that there exists a universal bundle $E$ on $X$, let $\mathbb{E}=\mathcal{E} n d^{0}(E)$. We will arrive at a subsheaf $\mathcal{G}_{E} \subset{ }^{\operatorname{tr}} \mathcal{A}_{E}^{-1}$ (see [2] for details of ${ }^{\operatorname{tr}} \mathcal{A}_{E}^{-1}$ ) fitting into an exact sequence

$$
0 \rightarrow \omega_{X / S} \rightarrow \mathcal{G}_{E} \stackrel{\text { res }}{\longrightarrow} \mathbb{E} \rightarrow 0,
$$

which induces, by taking the 2-nd symmetric tensor, the exact sequence

$$
0 \rightarrow \mathcal{G}_{E} \rightarrow S^{2}\left(\mathcal{G}_{E}\right) \otimes T_{X / S} \stackrel{\mathrm{Sym}^{2}(\mathrm{res}) \otimes \mathrm{id}}{\longrightarrow} S^{2}(\mathbb{E}) \otimes T_{X / S} \rightarrow 0 .
$$

Define $S\left(\mathcal{G}_{E}\right):=\left(\operatorname{Sym}^{2}(\mathrm{res}) \otimes \mathrm{id}\right)^{-1}\left(\mathrm{id} \otimes T_{X / S}\right)$, which fits into

$$
0 \rightarrow \mathcal{G}_{E} \stackrel{\iota}{\rightarrow} S\left(\mathcal{G}_{E}\right) \stackrel{q}{\rightarrow} T_{X / S} \rightarrow 0,
$$

where $q=\operatorname{Sym}^{2}($ res $) \otimes \mathrm{id}, \iota(\alpha)=\operatorname{Sym}^{2}(\alpha \otimes d t) \otimes \partial_{t}$ locally. Let

$$
\begin{aligned}
0 & \rightarrow R^{1} \pi_{*}\left(\omega_{X / S}\right) \rightarrow R^{1} \pi_{*}\left(\mathcal{G}_{E}\right) \stackrel{\text { res }}{\rightarrow} R^{1} \pi_{*}(\mathbb{E}) \rightarrow 0 \\
0 & \rightarrow R^{1} \pi_{*}\left(\mathcal{G}_{E}\right) \stackrel{\iota}{\rightarrow} R^{1} \pi_{*} S\left(\mathcal{G}_{E}\right) \stackrel{q}{\rightarrow} R^{1} \pi_{*}\left(T_{X / S}\right) \rightarrow 0
\end{aligned}
$$

be the exact sequences induced by (2.2), (2.3). Let $\Delta \subset X \times_{S} X$ be the diagonal, consider the induced diagram

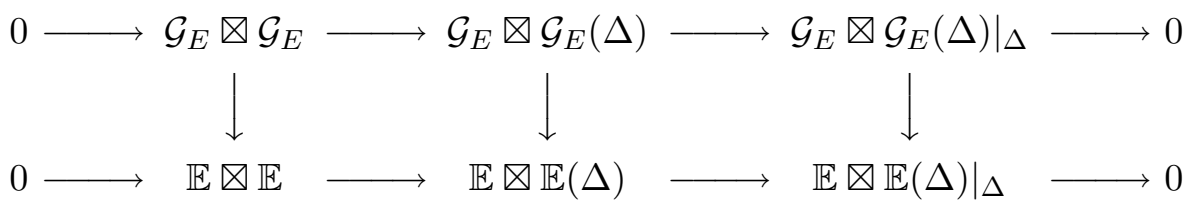


on $X \times_{S} X\left(\mathbb{E} \otimes \mathbb{E}\right.$ denotes $\left.p_{1}^{*} \mathbb{E} \otimes p_{2}^{*} \mathbb{E}\right)$. All vertical maps are induced by $\mathcal{G}_{E} \stackrel{\text { res }}{\longrightarrow} \mathbb{E} \rightarrow 0$, thus (2.3) is a subsequence of the rightmost vertical map above. Let $\mathcal{F}_{1} \subset \mathcal{G}_{E} \otimes \mathcal{G}_{E}(\Delta), \mathcal{F}_{2} \subset \mathbb{E} \otimes \mathbb{E}(\Delta)$ be subsheaves satisfying

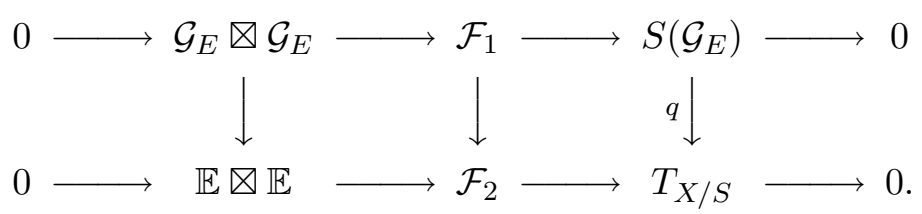

Taking direct images, considering the connecting maps, we have

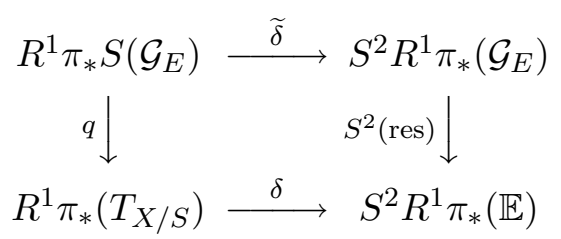

which induces the commutative diagram

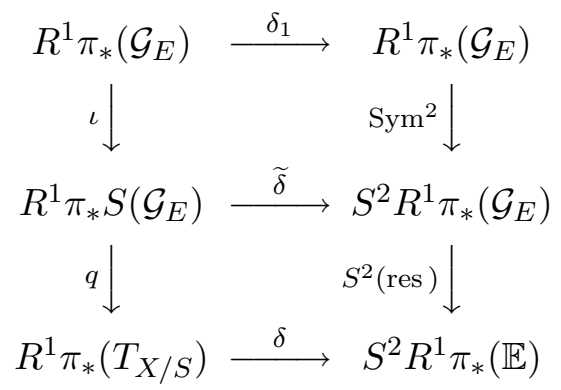

where $\delta_{1}$ is defined such that the diagram is commutative, the first vertical exact sequence is (2.5), the second vertical exact sequence is induced by taking the 2-nd symmetric tensor of (2.4) (note that $\mathcal{O}_{S} \cong$ $\left.R^{1} \pi_{*}\left(\omega_{X / S}\right)\right)$. For Sym ${ }^{2}$ see Remark 2.6.

Lemma 2.4. The map $\delta_{1}: R^{1} \pi_{*}\left(\mathcal{G}_{E}\right) \rightarrow R^{1} \pi_{*}\left(\mathcal{G}_{E}\right)$ is the identity map.

Proof. See Lemma 3.18.

q.e.d. 


\section{Theorem 2.5.}

i) There is an isomorhism $\phi: R^{1} \pi_{*}\left(\mathcal{G}_{E}\right) \cong \mathcal{D}_{S / \mathcal{M}}^{\leq 1}\left(\lambda_{\mathbb{E}}\right)$ such that the following diagram is commutative:

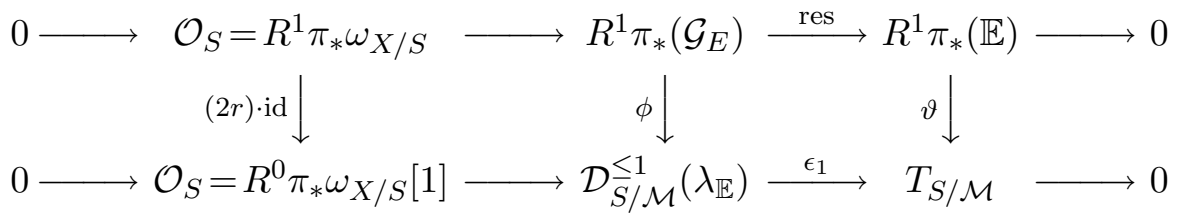

where $\vartheta$ is a Kodaira-Spencer type identification.

ii) For any affine covering $\left\{\mathcal{M}_{i}\right\}_{i \in I}$ of $\mathcal{M}$, on each $S_{i}:=f^{-1}\left(\mathcal{M}_{i}\right)$, there is an isomorphism $\phi_{i}: R^{1} \pi_{*} S\left(\mathcal{G}_{E}\right) \cong \mathcal{D}_{S}^{\leq 1}\left(\lambda_{\mathbb{E}}\right)$ such that

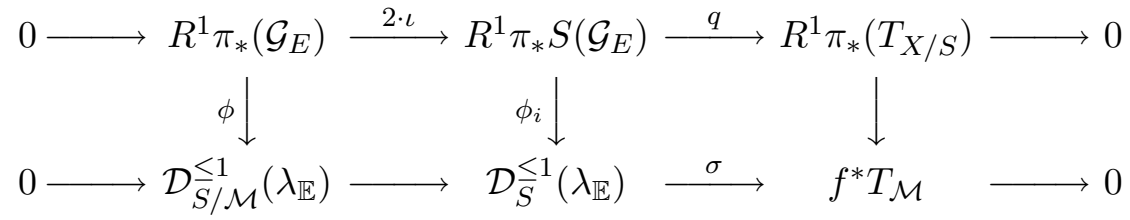

is commutative on $S_{i}$. Moreover, $\left\{\phi_{i}-\phi_{j}\right\}$ defines a class in $\mathrm{H}^{1}\left(\Omega_{\mathcal{M}}^{1}\right)$.

Proof. See Theorem 3.7, Corollary 3.12 and Remark 3.17. q.e.d.

By the above theorem, on each $S_{i}$, we get commutative diagram

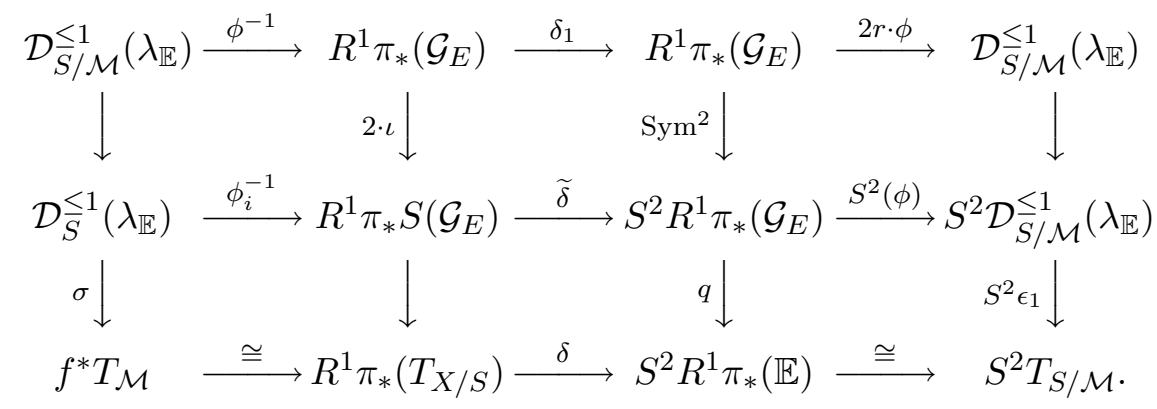

\section{Remark 2.6.}

i) For a precise definition of $\mathrm{Sym}^{2}$ above we refer to proof of Lemma 3.18.

ii) The insertion of $2 r$ in $2 r \cdot \phi$ in the first row is due to Theorem 2.5 i) combined with the definition of $\mathrm{Sym}^{2}$. 
The above diagram gives the following commutative diagram on $S_{i}$ :

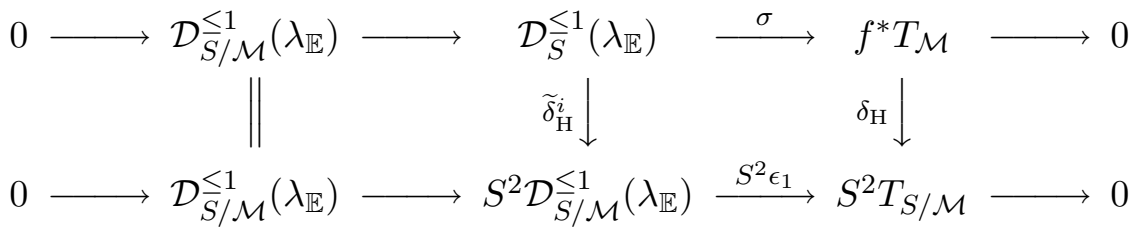

where $\delta_{\mathrm{H}}, \widetilde{\delta}_{\mathrm{H}}^{i}$ are defined in a clear way such that each $\widetilde{\delta}_{\mathrm{H}}^{i}$ induces an identity map on $\mathcal{D}_{S / \mathcal{M}}^{\leq 1}\left(\lambda_{\mathbb{E}}\right)$.

Proposition 2.7. Replace $\mathcal{M}$ by an affine open set. For any $\rho=$ $\delta_{\mathrm{H}}(v) \in H^{0}\left(S, S^{2} T_{S / \mathcal{M}}\right)$, where $0 \neq v \in H^{0}\left(S, f^{*} T_{\mathcal{M}}\right)$, one has:

i) $2 a(\mathcal{L}, \rho)=b(\mathcal{L}, \rho)+{ }^{t} b\left(\mathcal{L}^{-1} \otimes K_{S / \mathcal{M}}, \rho\right)$.

ii) When $\mathcal{L}=K_{S / \mathcal{M}}^{\mu}$, where $\mu \in \mathbb{Q}$ and $\mu \neq 1$, one has

$$
a\left(K_{S / \mathcal{M}}^{\mu}, \rho\right)=\frac{2 \mu-1}{2 \mu} b\left(K_{S / \mathcal{M}}^{\mu}, \rho\right) .
$$

Proof. To prove i), by Proposition 2.3 iii), it is enough to show that $c(\mathcal{K}, \rho)=0$. The class is independent of $\mathcal{L}$. Thus, by taking $\mathcal{L}=\mathcal{O}_{S}$ and using Proposition 2.3 ii), it is enough to show that

$$
{ }^{t} b(\mathcal{K}, \rho) \in H^{1}\left(\mathcal{D}_{S / \mathcal{M}}^{\leq 1}\left(\mathcal{O}_{S}\right)\right)=H^{1}\left(\mathcal{O}_{S}\right) \oplus H^{1}\left(T_{S / \mathcal{M}}\right)
$$

has trivial projection in $H^{1}\left(\mathcal{O}_{S}\right)$ where $\mathcal{K}=K_{S / \mathcal{M}}$. Using Theorem 2.5 (we need here that $\mathcal{M}$ be replaced by an affine open set), we have (noting $\left.\mathcal{K}=\lambda_{\mathbb{E}}\right)$

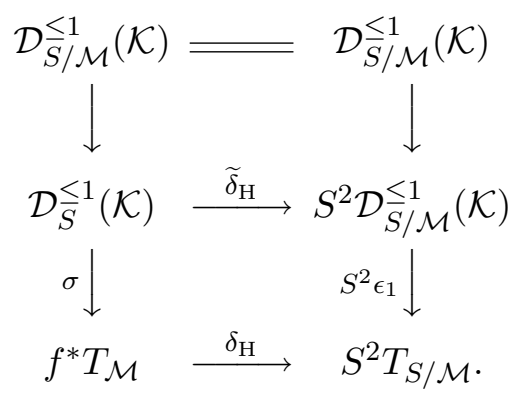

Let $\left\{\mathcal{U}_{i}\right\}_{i \in I}$ be an affine covering of $S$ and $v_{i} \in T_{S}\left(\mathcal{U}_{i}\right)$ be local liftings of $v \in f^{*} T_{\mathcal{M}}(S)$. Let $\left\{d_{i} \in \mathcal{D}_{S}^{\leq 1}(\mathcal{K})\left(\mathcal{U}_{i}\right)\right\}_{i \in I}$ be such that $\epsilon_{1}\left(d_{i}\right)=v_{i}(i \in$ $I)$. Then, by above diagram, $b(\mathcal{K}, \rho)=\left\{d_{i}-d_{j} \in \mathcal{D} \frac{\leq 1}{S / \mathcal{M}}(\mathcal{K})\left(\mathcal{U}_{i} \cap \mathcal{U}_{j}\right)\right\}$. Thus the class ${ }^{t} b(\mathcal{K}, \rho) \in H^{1}\left(\mathcal{D}_{S / \mathcal{M}}^{\leq 1}\left(\mathcal{O}_{S}\right)\right)=H^{1}\left(\mathcal{O}_{S}\right) \oplus H^{1}\left(T_{S / \mathcal{M}}\right)$ is defined by the cocycle $\left\{{ }^{t} d_{i}-{ }^{t} d_{j}\right\}$, where ${ }^{t} d_{i}:=A_{i}-v_{i} \in \mathcal{D}_{S}^{\leq 1}\left(\mathcal{O}_{S}\right)\left(\mathcal{U}_{i}\right)$. 
Thus the projection of ${ }^{t} b(\mathcal{K}, \rho)$ in $H^{1}\left(\mathcal{O}_{S}\right)$ is defined by $\left\{A_{i}-A_{j}\right\}$, which is a trivial class.

To show ii), we remark that for any nonzero $\mu \in \mathbb{Q}$, through canonical isomorphisms $\psi_{\mu}: \mathcal{D}^{\leq 1}(\mathcal{K}) \cong \mathcal{D}^{\leq 1}\left(\mathcal{K}^{\mu}\right)$, the above diagram induces

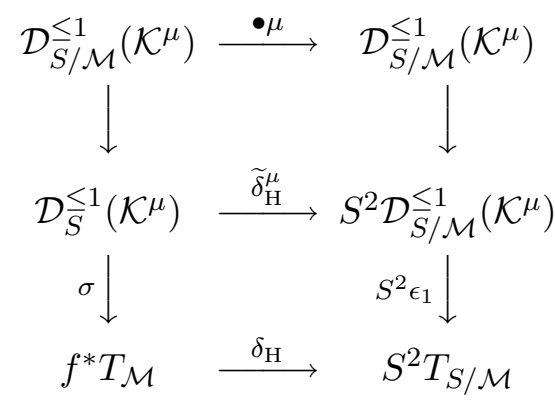

where $\widetilde{\delta}_{\mathrm{H}}^{\mu}=S^{2} \psi_{\mu} \circ \widetilde{\delta}_{\mathrm{H}} \circ \psi_{\mu}^{-1}$. Using the above diagram, we can compute ${ }^{t} b\left(\mathcal{K}^{1-\mu}, \rho\right)$. Let $\left\{d_{i} \in \mathcal{D}_{S}^{\leq 1}\left(\mathcal{K}^{1-\mu}\right)\left(\mathcal{U}_{i}\right)\right\}_{i \in I}$ be such that $\epsilon_{1}\left(d_{i}\right)=v_{i}$ $(i \in I)$. Then $b\left(\mathcal{K}^{1-\mu}, \rho\right)=(1-\mu)\left\{d_{i}-d_{j}\right\}$, which implies that

$$
{ }^{t} b\left(\mathcal{K}^{1-\mu}, \rho\right)=(1-\mu)\left\{{ }^{t} d_{i}-{ }^{t} d_{j}\right\} .
$$

On the other hand, $\left\{-{ }^{t} d_{i} \in \mathcal{D}_{S}^{\leq 1}\left(\mathcal{K}^{\mu}\right)\left(\mathcal{U}_{i}\right)\right\}_{i \in I}$ are local liftings of $v$, which means that $b\left(\mathcal{K}^{\mu}, \rho\right)=-\mu\left\{{ }^{t} d_{i}-{ }^{t} d_{j}\right\}=\frac{\mu}{\mu-1}{ }^{t} b\left(\mathcal{K}^{1-\mu}, \rho\right)$. Thus

$$
a\left(\mathcal{K}^{\mu}, \rho\right)=\frac{2 \mu-1}{2 \mu} b\left(\mathcal{K}^{\mu}, \rho\right) . \quad \text { q.e.d. }
$$

Theorem 2.8. Replace $\mathcal{M}$ by an affine open set, and let $\left\{\mathcal{U}_{i}\right\}_{i \in I}$ be an affine open covering of $S$. Then, for any $0 \neq v \in f^{*} T_{\mathcal{M}}(S)$, there are

$$
d_{S}^{i} \in \mathcal{D}_{\bar{S}}^{\leq 1}\left(\mathcal{K}^{\mu}\right)\left(\mathcal{U}_{i}\right), d_{S / \mathcal{M}}^{i} \in \mathcal{D}_{S / \mathcal{M}}^{\leq 1}\left(\mathcal{K}^{\mu}\right)\left(\mathcal{U}_{i}\right), D_{S / \mathcal{M}}^{i} \in \mathcal{D}_{S / \mathcal{M}}^{\leq 2}\left(\mathcal{K}^{\mu}\right)\left(\mathcal{U}_{i}\right),
$$

where $\mathcal{K}:=K_{S / \mathcal{M}}$, such that

$$
\left\{\mathrm{H}(v)_{i}:=d_{S}^{i}-d_{S / \mathcal{M}}^{i}+\frac{2}{1-2 \mu} D_{S / \mathcal{M}}^{i} \in \mathcal{D}_{\bar{S}}^{\leq 2}\left(\mathcal{K}^{\mu}\right)\left(\mathcal{U}_{i}\right)\right\}_{i \in I}
$$

form a global section $\mathrm{H}(v) \in H^{0}\left(S, \mathcal{D}_{\bar{S}}^{\leq 2}\left(\mathcal{K}^{\mu}\right)\right)$ with

$$
\sigma(\mathrm{H}(v))=v, \quad \epsilon_{2}(\mathrm{H}(v))=\frac{2}{1-2 \mu} \delta_{\mathrm{H}}(v) .
$$

Proof. Let $\left\{d_{S}^{i} \in \mathcal{D}_{S}^{\leq 1}\left(\mathcal{K}^{\mu}\right)\left(\mathcal{U}_{i}\right)\right\}_{i \in I}$ be such that $\sigma\left(d_{S}^{i}\right)=\left.v\right|_{\mathcal{U}_{i}}$. Then

$$
\left\{\mu\left(d_{S}^{i}-d_{S}^{j}\right) \in \mathcal{D}_{S / \mathcal{M}}^{\leq 1}\left(\mathcal{K}^{\mu}\right)\left(\mathcal{U}_{i} \cap \mathcal{U}_{j}\right)\right\}
$$


defines the class $b\left(\mathcal{K}^{\mu}, \delta_{\mathrm{H}}(v)\right) \in H^{1}\left(S, \mathcal{D}_{S / \mathcal{M}}^{\leq 1}\left(\mathcal{K}^{\mu}\right)\right)$, which is the obstruction for lifting $\delta_{\mathrm{H}}(v) \in H^{0}\left(S, S^{2} T_{S / \mathcal{M}}\right)$ to $H^{0}\left(S, S^{2} \mathcal{D}_{S / \mathcal{M}}^{\leq 1}\left(\mathcal{K}^{\mu}\right)\right)$. Let

$$
\left\{D_{S / \mathcal{M}}^{i} \in \mathcal{D}_{S / \mathcal{M}}^{\leq 2}\left(\mathcal{K}^{\mu}\right)\left(\mathcal{U}_{i}\right)\right\}_{i \in I}
$$

be local liftings of $\delta_{\mathrm{H}}(v)$. Then, by Proposition 2.7,

$$
\left\{d_{S}^{i}-d_{S}^{j}\right\}=\frac{2}{2 \mu-1}\left\{D_{S / \mathcal{M}}^{i}-D_{S / \mathcal{M}}^{j}\right\}
$$

as cohomology classes. Thus there are $\left\{d_{S / \mathcal{M}}^{i} \in \mathcal{D}_{S / \mathcal{M}}^{\leq 1}\left(\mathcal{K}^{\mu}\right)\left(\mathcal{U}_{i}\right)\right\}_{i \in I}$ satisfying the requirements in the theorem.

Corollary 2.9. There exists a unique projective heat operator,

$$
\mathrm{H}: T_{\mathcal{M}} \rightarrow f_{*} \mathcal{W}\left(\Theta^{k}\right) / \mathcal{O}_{\mathcal{M}}
$$

(where $\mathcal{M}$ is global) such that $\left(f_{*} \epsilon_{2}\right) \cdot \mathrm{H}: T_{\mathcal{M}} \rightarrow f_{*} S^{2} T_{S / \mathcal{M}}$ coincides with $f_{*} \delta_{\mathrm{H}}$.

Proof. For any open set $U \subset \mathcal{M}$ and $v \in T_{\mathcal{M}}(U)$, by Theorem 2.8, we can construct a $\mathrm{H}(v) \in f_{*} \mathcal{W}\left(\Theta^{k}\right)(U)$. If $\mathrm{H}(v)^{\prime}$ is another such operator, $\mathrm{H}(v)-\mathrm{H}(v)^{\prime}$ must have symbol in $H^{0}\left(f^{-1}(U), T_{S / \mathcal{M}}\right)=0$, so

$$
\mathrm{H}(v)-\mathrm{H}(v)^{\prime} \in H^{0}\left(f^{-1}(U), \mathcal{O}_{S}\right)=f_{*} \mathcal{O}_{S}(U)=\mathcal{O}_{\mathcal{M}}(U) .
$$

Hence a unique map $T_{\mathcal{M}} \rightarrow f_{*} \mathcal{W}\left(\Theta^{k}\right) / \mathcal{O}_{\mathcal{M}}$.

q.e.d.

Now we construct the logarithmic extension of above operator. Let $\widetilde{\mathcal{C}} \rightarrow \widetilde{\mathcal{M}}$ be the family in the introduction. Let $\mathcal{U}(r, d) \rightarrow \widetilde{\mathcal{M}}$ be the family of moduli spaces of semistable (for canonical polarization) torsionfree sheaves of rank $r$ and degree $d$. Fix a line bundle $\mathcal{N}$ on $\widetilde{\mathcal{C}}$ of relative degree $d$, let $f: S \rightarrow \mathcal{M}$ be the family of moduli spaces of stable bundles of rank $r$ with fixed determinant $\left.\mathcal{N}\right|_{C}([C] \in \mathcal{M})$.

Notation 2.10. Let $f_{Z}: Z \rightarrow \widetilde{\mathcal{M}}$ be defined as the Zariski closure of $S$ in $\mathcal{U}(r, d)$ and $f_{T}: T \rightarrow \widetilde{\mathcal{M}}$ be the open set of $Z$ consisting of locally free sheaves. Let $\widetilde{f}: \widetilde{S} \subset T \rightarrow \widetilde{\mathcal{M}}$ be the open set of stable bundles. Then $\widetilde{f}: \widetilde{S} \rightarrow \widetilde{\mathcal{M}}$ is smooth (cf. Lemma 4.4).

Let $E$ be the unversal bundle on $\widetilde{X}$, where $\widetilde{X}$ is defined by

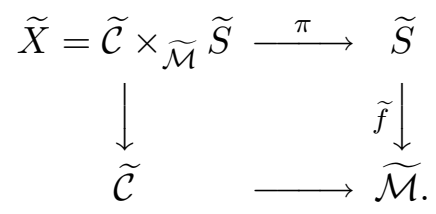


Let $D \subset \widetilde{X}$ be the divisor of singular curves. Then we have

$$
\mathcal{G}_{E} \subset{ }^{\operatorname{tr}} \mathcal{A}_{E}^{-1}(\log D)
$$

fitting into the exact sequence

$$
0 \rightarrow \omega_{\widetilde{X} / \widetilde{S}} \rightarrow \mathcal{G}_{E} \stackrel{\text { res }}{\longrightarrow} \mathbb{E}:=\mathcal{E} n d^{0}(E) \rightarrow 0
$$

Similarly, there is a sheaf $S\left(\mathcal{G}_{E}\right) \subset \operatorname{Sym}^{2}\left(\mathcal{G}_{E}\right) \otimes \omega_{\widetilde{X} / \widetilde{S}}^{-1}$ fitting into

$$
0 \rightarrow \mathcal{G}_{E} \stackrel{\iota}{\rightarrow} S\left(\mathcal{G}_{E}\right) \stackrel{q}{\rightarrow} \omega_{\widetilde{X} / \widetilde{S}}^{-1} \rightarrow 0 .
$$

They induce the following exact sequences:

$$
\begin{aligned}
0 & \rightarrow R^{1} \pi_{*}\left(\omega_{\tilde{X} / \widetilde{S}}\right) \rightarrow R^{1} \pi_{*}\left(\mathcal{G}_{E}\right) \stackrel{\text { res }}{\longrightarrow} R^{1} \pi_{*}(\mathbb{E}) \rightarrow 0, \\
0 & \rightarrow R^{1} \pi_{*}\left(\mathcal{G}_{E}\right) \stackrel{\iota}{\rightarrow} R^{1} \pi_{*} S\left(\mathcal{G}_{E}\right) \stackrel{q}{\rightarrow} R^{1} \pi_{*}\left(\omega_{\widetilde{X} / \widetilde{S}}^{-1}\right) \rightarrow 0 .
\end{aligned}
$$

Let $\widetilde{\Delta} \subset \widetilde{X} \times_{\widetilde{S}} \widetilde{X}:=P$ be the diagonal and $\mathcal{O}(\widetilde{\Delta})$ be the dual of its ideal sheaf. Then $0 \rightarrow \omega_{P / \widetilde{S}} \rightarrow \omega_{P / \widetilde{S}}(\widetilde{\Delta}) \rightarrow \mathcal{E}_{x} t_{P}^{1}\left(\mathcal{O}_{\widetilde{\Delta}}, \omega_{P / \widetilde{S}}\right) \rightarrow 0$, one checks that the relative dualizing sheaf $\omega_{P / \widetilde{S}}$ is $\omega_{\widetilde{X} / \widetilde{S}} \bigotimes \omega_{\widetilde{X} / \widetilde{S}}$ and $\mathcal{E}_{x} t_{P}^{1}\left(\mathcal{O}_{\widetilde{\Delta}}, \omega_{P / \widetilde{S}}\right)$ is the relative dualizing sheaf of $\widetilde{\Delta} / \widetilde{S}$. Thus we have

$$
0 \rightarrow \mathcal{O} \rightarrow \mathcal{O}(\widetilde{\Delta}) \rightarrow \omega_{\widetilde{X} / \widetilde{S}}^{-1} \rightarrow 0,
$$

which similarly induces the commutative diagram

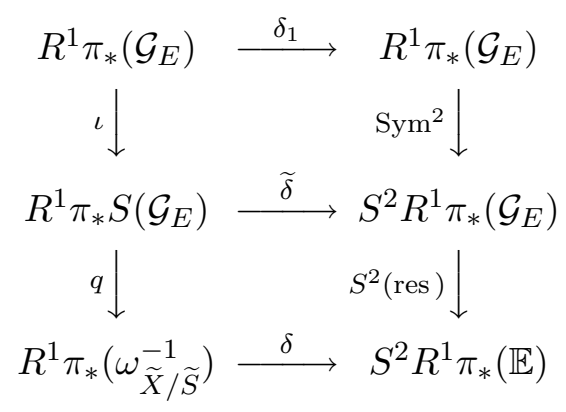

where $\delta_{1}$ denote the map induced by $\widetilde{\delta}$, the first vertical exact sequence is (2.9), the second vertical exact sequence is induced by taking the 2 -nd symmetric tensor of $(2.8)$ (note that $\mathcal{O}_{\widetilde{S}} \cong R^{1} \pi_{*}\left(\omega_{\widetilde{X} / \widetilde{S}}\right)$ ). 
Let $B=\widetilde{\mathcal{M}} \backslash \mathcal{M}$ and $W=\widetilde{f}^{-1}(B) \subset \widetilde{S}$. Consider

$$
\begin{aligned}
0 & \rightarrow T_{\widetilde{S} / \widetilde{\mathcal{M}}} \rightarrow T_{\widetilde{S}} \stackrel{d \widetilde{f}}{\longrightarrow} \widetilde{f}^{*} T_{\widetilde{\mathcal{M}}} \rightarrow 0, \\
0 \rightarrow \mathcal{D}_{\widetilde{S} / \widetilde{\mathcal{M}}}^{\leq 1}(\mathcal{L}) \rightarrow \mathcal{D} & \stackrel{\underline{\widetilde{S}}}{\leq 1}(\mathcal{L}) \stackrel{\sigma}{\rightarrow} \widetilde{f}^{*} T_{\widetilde{\mathcal{M}}} \rightarrow 0 .
\end{aligned}
$$

Notation 2.11. Let $T_{\widetilde{\mathcal{M}}}(B) \subset T_{\mathcal{M}}$ be the subsheaf of vector fields that preserve $B$. Let $T_{\widetilde{S}}(\log W) \subset T_{\widetilde{S}}, \mathcal{D} \underset{\widetilde{S}}{\leq 1}(\mathcal{L})(\log W) \subset \mathcal{D} \underset{\widetilde{S}}{\leq 1}(\mathcal{L})$ be the subsheaves such that the following are exact sequences:

$$
\begin{aligned}
0 & \rightarrow T_{\widetilde{S} / \widetilde{\mathcal{M}}} \rightarrow T_{\widetilde{S}}(\log W) \stackrel{d \widetilde{f}}{\rightarrow} \widetilde{f}^{*} T_{\widetilde{\mathcal{M}}}(B) \rightarrow 0, \\
0 & \rightarrow \mathcal{D} \underset{\widetilde{S} / \widetilde{\mathcal{M}}}{\leq 1}(\mathcal{L}) \rightarrow \mathcal{D} \widetilde{\widetilde{S}}_{\tilde{S}}^{\leq 1}(\mathcal{L})(\log W) \stackrel{\sigma}{\rightarrow} \widetilde{f}^{*} T_{\widetilde{\mathcal{M}}}(B) \rightarrow 0 .
\end{aligned}
$$

Lemma 2.12. The map $\delta_{1}: R^{1} \pi_{*}\left(\mathcal{G}_{E}\right) \rightarrow R^{1} \pi_{*}\left(\mathcal{G}_{E}\right)$ is identity.

Proof. Since $S \subset \widetilde{S}$ is dense in $\widetilde{S}$ by definition (cf. Notation 2.10), the lemma follows from Lemma 2.4.

q.e.d.

\section{Theorem 2.13.}

i) There is an isomorhism $\phi: R^{1} \pi_{*}\left(\mathcal{G}_{E}\right) \cong \mathcal{D} \underset{\widetilde{S} / \widetilde{\mathcal{M}}}{\leq 1}\left(\lambda_{\mathbb{E}}\right)$ such that the following diagram is commutative:

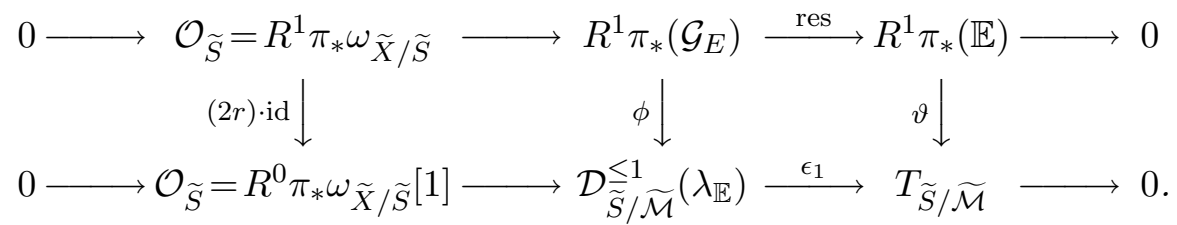

ii) For any affine covering $\left\{\widetilde{\mathcal{M}}_{i}\right\}_{i \in I}$ of $\widetilde{\mathcal{M}}$, on each $\widetilde{S}_{i}:=\widetilde{f}^{-1}\left(\widetilde{\mathcal{M}}_{i}\right)$, there is an isomorphism $\phi_{i}: R^{1} \pi_{*} S\left(\mathcal{G}_{E}\right) \cong \mathcal{D} \underset{\widetilde{S}}{\leq 1}\left(\lambda_{\mathbb{E}}\right)(\log W)$ such that the following diagram commutes on $\widetilde{S}_{i}$

$0 \longrightarrow R^{1} \pi_{*}\left(\mathcal{G}_{E}\right) \stackrel{2 \cdot \iota}{\longrightarrow} R^{1} \pi_{*} S\left(\mathcal{G}_{E}\right) \stackrel{q}{\longrightarrow} R^{1} \pi_{*}\left(\omega_{\tilde{X} / \widetilde{S}}^{-1}\right) \longrightarrow 0$

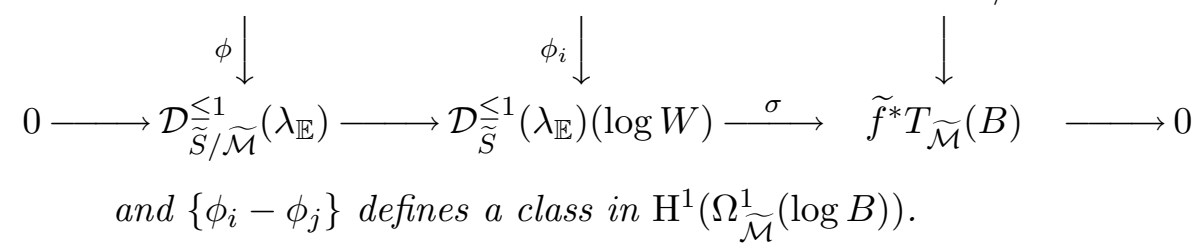

Proof. See Proposition 4.5 and Theorem 4.8. $\quad$ q.e.d. 
Similarly, we have the commutative diagram on each $\widetilde{S}_{i}$

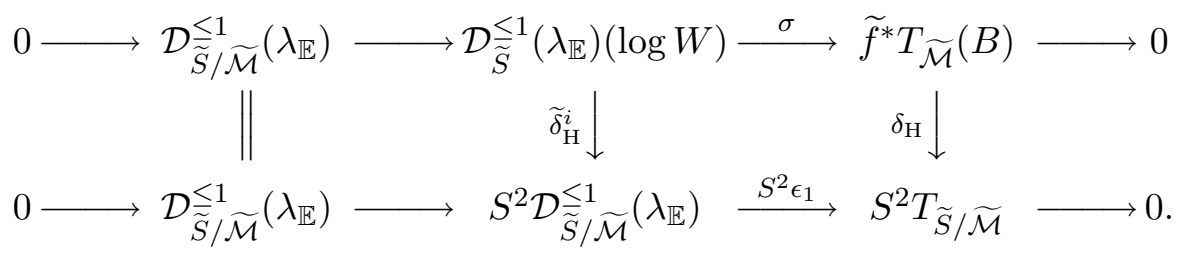

Proposition 2.14. For any $\rho=\delta_{\mathrm{H}}(v) \in H^{0}\left(\widetilde{S}, S^{2} T_{\widetilde{S} / \widetilde{\mathcal{M}}}\right)$, where $\widetilde{\mathcal{M}}$ is replaced by an affine open set and $v \in H^{0}\left(\widetilde{S}, \widetilde{f}^{*} T_{\widetilde{\mathcal{M}}}(B)\right)$, one has:

i) $2 a(\mathcal{L}, \rho)=b(\mathcal{L}, \rho)+{ }^{t} b\left(\mathcal{L}^{-1} \otimes K_{\widetilde{S} / \widetilde{\mathcal{M}}}, \rho\right)$.

ii) When $\mathcal{L}=K_{\widetilde{S} / \widetilde{\mathcal{M}}}^{\mu}$, where $\mu \in \mathbb{Q}$ and $\mu \neq 1$, one has

$$
a\left(K_{\widetilde{S} / \widetilde{\mathcal{M}}}^{\mu}, \rho\right)=\frac{2 \mu-1}{2 \mu} b\left(K_{\widetilde{S} / \widetilde{\mathcal{M}}}^{\mu}, \rho\right) .
$$

Proof. Note that we still have $\mathcal{K}=K_{\widetilde{S} / \widetilde{\mathcal{M}}}=\lambda_{\mathbb{E}}$, the proof is the same as that of Proposition 2.7 by using Theorem 2.13. We just remark two points:

(1) For any operator $d$ of $\mathcal{D} \underset{\widetilde{S}}{\leq 1}(\mathcal{L})(\log W) \subset \mathcal{D} \underset{\widetilde{S}}{\leq 1}(\mathcal{L})$, its adjoint operator ${ }^{t} d$ is still in $\mathcal{D} \underset{\widetilde{S}}{\leq 1}\left(\mathcal{L}^{-1} \otimes \mathcal{K}\right)(\log W)$.

(2) For any nonzero $\mu \in \mathbb{Q}$, the canonical isomorphism $\psi_{\mu}: \mathcal{D} \underset{\widetilde{S}}{\leq 1}(\mathcal{K})$ $\cong \mathcal{D} \underset{\widetilde{S}}{\leq}\left(\mathcal{K}^{\mu}\right)$ induces an isomorphism $\mathcal{D}_{\widetilde{S}}^{\leq 1}(\mathcal{K})(\log W) \cong$ $\mathcal{D} \underset{\widetilde{S}}{\leq 1}\left(\mathcal{K}^{\mu}\right)(\log W) . \quad$ q.e.d.

Theorem 2.15. Replace $\widetilde{\mathcal{M}}$ by an affine open set, and let $\left\{\mathcal{U}_{i}\right\}_{i \in I}$ be an affine open covering of $\widetilde{S}$. Then, for any $v \in \widetilde{f}^{*} T_{\widetilde{\mathcal{M}}}(B)(\widetilde{S})$, there are

$$
d_{\widetilde{S}}^{i} \in \mathcal{D} \underset{\widetilde{S}}{\leq 1}\left(\mathcal{K}^{\mu}\right)(\log W)\left(\mathcal{U}_{i}\right), \quad d_{\widetilde{S} / \widetilde{\mathcal{M}}}^{i} \in \mathcal{D} \underset{\widetilde{S} / \widetilde{\mathcal{M}}}{\leq 1}\left(\mathcal{K}^{\mu}\right)\left(\mathcal{U}_{i}\right),
$$

and $D_{\widetilde{S} / \widetilde{\mathcal{M}}}^{i} \in \mathcal{D} \underset{\widetilde{S} / \widetilde{\mathcal{M}}}{\leq 2}\left(\mathcal{K}^{\mu}\right)\left(\mathcal{U}_{i}\right)$ such that

$$
\left\{\mathrm{H}(v)_{i}:=d_{\widetilde{S}}^{i}-d_{\widetilde{S} / \widetilde{\mathcal{M}}}^{i}+\frac{2}{1-2 \mu} D_{\widetilde{S} / \widetilde{\mathcal{M}}}^{i} \in \mathcal{D} \underset{\widetilde{S}}{\leq 2}\left(\mathcal{K}^{\mu}\right)\left(\mathcal{U}_{i}\right)\right\}_{i \in I}
$$

form a global section $\mathrm{H}(v) \in H^{0}\left(\widetilde{S}, \mathcal{D} \underset{\widetilde{S}}{\leq 2}\left(\mathcal{K}^{\mu}\right)\right)$ with

$$
\sigma(\mathrm{H}(v))=v, \quad \epsilon_{2}(\mathrm{H}(v))=\frac{2}{1-2 \mu} \delta_{\mathrm{H}}(v) .
$$



Then

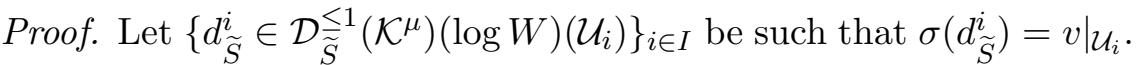

$$
\left\{\mu\left(d_{\widetilde{S}}^{i}-d_{\widetilde{S}}^{j}\right) \in \mathcal{D} \underset{\widetilde{S} / \widetilde{\mathcal{M}}}{\leq 1}\left(\mathcal{K}^{\mu}\right)\left(\mathcal{U}_{i} \cap \mathcal{U}_{j}\right)\right\}
$$

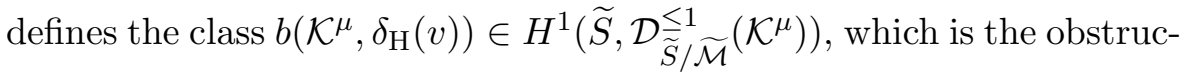
tion for lifting $\delta_{\mathrm{H}}(v) \in H^{0}\left(\widetilde{S}, S^{2} T_{\widetilde{S} / \widetilde{\mathcal{M}}}\right)$ to $H^{0}\left(\widetilde{S}, S^{2} \mathcal{D} \underset{\widetilde{S} / \widetilde{\mathcal{M}}}{\leq 1}\left(\mathcal{K}^{\mu}\right)\right.$. Let

$$
\left\{D_{\widetilde{S} / \widetilde{\mathcal{M}}}^{i} \in \mathcal{D} \underset{\widetilde{S} / \widetilde{\mathcal{M}}}{\leq 2}\left(\mathcal{K}^{\mu}\right)\left(\mathcal{U}_{i}\right)\right\}_{i \in I}
$$

be local liftings of $\delta_{\mathrm{H}}(v)$. Then, by Proposition 2.14,

$$
\left\{d_{\widetilde{S}}^{i}-d_{\widetilde{S}}^{j}\right\}=\frac{2}{2 \mu-1}\left\{D_{\widetilde{S} / \widetilde{\mathcal{M}}}^{i}-D_{\widetilde{S} / \widetilde{\mathcal{M}}}^{j}\right\}
$$

as cohomology classes. Thus there are $\left\{d_{\widetilde{S} / \widetilde{\mathcal{M}}}^{i} \in \mathcal{D} \underset{\widetilde{S} / \widetilde{\mathcal{M}}}{\leq 1}\left(\mathcal{K}^{\mu}\right)\left(\mathcal{U}_{i}\right)\right\}_{i \in I}$ satisfying the requirements in the theorem. q.e.d.

Corollary 2.16. There exists a unique projective heat operator

$$
\widetilde{\mathrm{H}}: T_{\widetilde{\mathcal{M}}}(B) \rightarrow \widetilde{f}_{*} \mathcal{D} \underset{\widetilde{S}}{\leq 2}\left(\Theta^{k}\right) / \mathcal{O}_{\widetilde{\mathcal{M}}}
$$

such that $\left(\widetilde{f}_{*} \epsilon_{2}\right) \cdot \widetilde{\mathrm{H}}: T_{\widetilde{\mathcal{M}}}(B) \rightarrow \widetilde{f}_{*} S^{2} T_{\widetilde{S} / \widetilde{\mathcal{M}}}$ coincides with $\widetilde{f}_{*} \delta_{\mathrm{H}}$. Moreover, $\widetilde{f}_{*}\left(\Theta^{k}\right)$ is a coherent sheaf on $\widetilde{\mathcal{M}}$.

Proof. The coherence of $\widetilde{f}_{*}\left(\Theta^{k}\right)$ follows from Theorem 4.10. Since $\widetilde{f}_{*} T_{\widetilde{S} / \widetilde{\mathcal{M}}}=0$ is still true, the rest follows the same proof of Corollary 2.9 if $\widetilde{f}_{*} \mathcal{O}_{\widetilde{S}}=\mathcal{O}_{\widetilde{\mathcal{M}}}$. We will prove (cf. Proposition 4.9) that the fibres of $\widetilde{f}: \widetilde{S} \rightarrow \widetilde{\mathcal{M}}$ is dense in the fibres of $f_{Z}: Z \rightarrow \widetilde{\mathcal{M}}$. Thus $Z \backslash \widetilde{S}$ has codimension at least 2 . By passing to the normalization $\iota: \widetilde{Z} \rightarrow Z$, $\iota^{-1}(\widetilde{S}) \cong \widetilde{S}$ and $\operatorname{codim}\left(\widetilde{Z} \backslash \iota^{-1}(\widetilde{S})\right) \geq 2$ since $\widetilde{S}$ is the open set of smooth points of $Z$ and $\iota$ is finite. We have $\widetilde{f}_{*} \mathcal{O}_{\widetilde{S}}=\left(f_{Z} \cdot \iota\right)_{*} \mathcal{O}_{\widetilde{Z}}=\mathcal{O}_{\widetilde{\mathcal{M}}}$. q.e.d.

\section{First order differential operators of the determinant bundle}

We give at first a short review of what we need from [2]. Let $\pi: X \rightarrow$ $S$ be a smooth proper morphism of relative dimension 1 between smooth varieties in characteristic 0 . We write $K_{X / S}$ or $\omega_{X / S}$ interchangeably for the dualizing sheaf. One has an exact sequence

$$
0 \rightarrow T_{X / S} \rightarrow T_{X} \stackrel{d \pi}{\longrightarrow} \pi^{*} T_{S} \rightarrow 0 .
$$


As in [2], one defines the subsheaf $\pi^{-1} T_{S} \subset \pi^{*} T_{S}$ and its preimage $T_{\pi}=d \pi^{-1} T_{\pi} \subset T_{X}$, defining the exact sequence

$$
0 \rightarrow T_{X / S} \rightarrow T_{\pi} \stackrel{d \pi}{\longrightarrow} \pi^{-1} T_{S} \rightarrow 0 .
$$

Let $E$ be a vector bundle on $X$, and $\lambda_{E}=\operatorname{det} R \pi_{*} E$ be its determinant bundle. The Atiyah algebra $\mathcal{A}_{E}$ is the subalgebra of the sheaf of firstorder differential operators on $E$ with symbolic part in (id $\left.\otimes T_{X}\right) \cong T_{X}$. The relative Atiyah algebra $\mathcal{A}_{E / S} \subset \mathcal{A}_{E}$ consists of those differential operators with symbolic part in $T_{X / S}$, and $\mathcal{A}_{E, \pi} \subset \mathcal{A}_{E}$ with symbolic part in $T_{\pi}$. Let ${ }^{\operatorname{tr}} \mathcal{A}_{E}^{-1}$ be the subquotient of the sheaf defined in [2]

$$
E \otimes_{\mathcal{O}_{S}}\left(E^{*} \otimes \omega_{X / S}\right)(2 \Delta) / E \otimes_{\mathcal{O}_{S}}\left(E^{*} \otimes \omega_{X / S}\right)(-\Delta)
$$

where $\Delta \subset X \times_{S} X$ denotes the diagonal, which fits into an exact sequence

$$
0 \rightarrow \omega_{X / S} \rightarrow{ }^{\operatorname{tr}} \mathcal{A}_{E}^{-1} \stackrel{\text { res }}{\longrightarrow} \mathcal{A}_{E / S} \rightarrow 0 .
$$

The trace complex is defined by

$$
{ }^{\operatorname{tr}} \mathcal{A}_{E}^{\bullet}: \mathcal{O}_{X} \stackrel{d_{X / S}}{\longrightarrow}{ }^{\operatorname{tr}} \mathcal{A}_{E}^{-1} \stackrel{\text { res }}{\longrightarrow} \mathcal{A}_{E, \pi}
$$

with $\mathcal{A}_{E, \pi}$ in degree 0 . One has:

Proposition 3.1. ${ }^{\text {tr }} \mathcal{A}_{E}^{\bullet}$ carries an algebra structure for which $R^{0} \pi_{*}\left({ }^{\operatorname{tr}} \mathcal{A}_{E}^{\bullet}\right)$ is canonically isomorphic to $\mathcal{A}_{\lambda_{E}}([\mathbf{2}], 2.3 .1$, see also [4]).

For the purpose of this paper it is more convenient to define the trace complex concentrated only on $i=-1$ and $i=0$ of the original trace complex. This modified trace complex is still denoted by ${ }^{\operatorname{tr}} \mathcal{A}_{E}^{\bullet}$ whose 0 -th direct image is easily seen to be the same as that of the original one. With the modified trace complex, one has now an exact sequence

$$
0 \rightarrow \omega_{X / S}[1] \rightarrow{ }^{\operatorname{tr}} \mathcal{A}_{E}^{\bullet} \rightarrow \mathcal{A}_{E, \pi}^{\bullet} \rightarrow 0,
$$

where the complex $\mathcal{A}_{E, \pi}^{\bullet}$ is defined by

$$
\mathcal{A}_{E, \pi}^{\bullet}: \mathcal{A}_{E / S} \rightarrow \mathcal{A}_{E, \pi},
$$

and thus is quasi-isomorphic to $\pi^{-1} T_{S}$.

Notation 3.2. Let $\pi: X \rightarrow S$ be as before, and $f: S \rightarrow M$ be a smooth morphism where $M$ is a smooth variety. Denote by

$$
\mathcal{A}_{E, \pi / \mathcal{M}} \subset \mathcal{A}_{E, \pi}, \quad T_{\pi / \mathcal{M}} \subset T_{X / \mathcal{M}} \subset T_{\pi}
$$

the pullback of $\pi^{-1} T_{S / \mathcal{M}} \subset \pi^{-1} T_{S}$. Let

$$
{ }^{\operatorname{tr}} \mathcal{A}_{E / \mathcal{M}}^{\bullet}:=\left({ }^{\operatorname{tr}} \mathcal{A}_{E}^{-1} \rightarrow \mathcal{A}_{E, \pi / \mathcal{M}}\right), \quad \mathcal{A}_{E, \pi / \mathcal{M}}^{\bullet}:=\left(\mathcal{A}_{E / S} \rightarrow \mathcal{A}_{E, \pi / \mathcal{M}}\right) .
$$


Proposition 3.3 ([2], Theorem 2.3.1). The exact sequences

$$
\begin{gathered}
0 \rightarrow \omega_{X / S}[1] \rightarrow{ }^{\operatorname{tr}} \mathcal{A}_{E}^{\bullet} \rightarrow \mathcal{A}_{E, \pi}^{\bullet} \rightarrow 0 \\
0 \rightarrow \omega_{X / S}[1] \rightarrow{ }^{\operatorname{tr}} \mathcal{A}_{E / M}^{\bullet} \rightarrow \mathcal{A}_{E, \pi / M}^{\bullet} \rightarrow 0
\end{gathered}
$$

have 0-th direct images (via $\pi$ ) isomorphic to

$$
\begin{gathered}
0 \rightarrow \mathcal{O}_{S} \rightarrow \mathcal{D}_{S}^{\leq 1}\left(\lambda_{E}\right) \rightarrow T_{S} \rightarrow 0 \\
0 \rightarrow \mathcal{O}_{S} \rightarrow \mathcal{D}_{S / M}^{\leq 1}\left(\lambda_{E}\right) \rightarrow T_{S / M} \rightarrow 0 .
\end{gathered}
$$

Furthermore, we need to review the description of tr $\mathcal{A}_{E}^{\bullet}$ in terms of local coordinates, [2], p. 660. Let $t$ be a local coordinate (along the fiber), and a trivilization $I: \mathcal{O}_{X}^{n} \cong E$; $s$ a local coordinate on $S$. Note $t$ naturally induces local coordinates $\left(t_{1}, t_{2}\right)$ around the diagonal of $X \times{ }_{S} X$. One has isomorphisms via $t$ and $I$,

$$
\begin{aligned}
(t, I)_{-1} & : \mathcal{O}_{X} \oplus \operatorname{Mat}_{n}\left(\mathcal{O}_{X}\right) \oplus \mathcal{O}_{X} \\
& \cong{ }^{\operatorname{tr}} \mathcal{A}_{E}^{-1},(t, I)_{-1}(\chi, B, \nu)=(\chi, B, \nu)_{(t, I)} \\
& =I\left[\frac{\chi\left(t_{1}\right)}{\left(t_{2}-t_{1}\right)^{2}}+\frac{B\left(t_{1}\right)}{\left(t_{2}-t_{1}\right)}+\nu\left(t_{1}\right)\right] d t_{2} ; \\
(t, I)_{0} & : T_{\pi} \oplus \operatorname{Mat}_{n}\left(\mathcal{O}_{X}\right) \\
& \cong{ }^{\operatorname{tr}} \mathcal{A}_{E}^{0}=\mathcal{A}_{E, \pi},(t, I)_{0}(\tau, A) \\
& =(\tau, A)_{I}=(t, I)_{0}\left[\tau(t, s) \partial_{t}+\mu(s) \partial_{s}+A\right] .
\end{aligned}
$$

For different choices of coordinates and trivializations, one has the gauge change and coordinate change formulas: with $g \in \operatorname{GL}_{r}\left(\mathcal{O}_{X}\right)$ and $y=$ $y(t)$ (the notation below being slightly different from that of $[\mathbf{2}]$ )

$$
\begin{gathered}
(\tau, A):=\alpha \stackrel{g}{\longrightarrow}\left(\tau,-\tau(g) g^{-1}+g A g^{-1}\right):=\beta\left(\Leftrightarrow \alpha_{I g}=\beta_{I}\right) ; \\
(\chi, B, \nu) \stackrel{g}{\longrightarrow}\left(\chi,-\chi g^{\prime} g^{-1}+g B g^{-1}\right. \\
\left.\operatorname{Tr}\left(-\frac{1}{2} \chi g^{\prime \prime} g^{-1}+\chi\left(g^{\prime} g^{-1}\right)^{2}-B g^{-1} g^{\prime}\right)+\nu\right) ; \\
(\chi, B, \nu) \stackrel{y(t)}{\longrightarrow}\left(\chi y^{\prime-1}, B, r \chi\left(\frac{1}{6} \frac{y^{\prime \prime \prime}}{y^{\prime 2}}-\frac{1}{4} \frac{y^{\prime \prime 2}}{y^{\prime 3}}\right)+\frac{1}{2} \frac{y^{\prime \prime}}{y^{\prime}} \operatorname{Tr} B+\nu y^{\prime}\right) .
\end{gathered}
$$

The main result of this section is Theorem 3.7 which enables us to take care of Theorem 2.5. Following the notation of Section 2, let

$$
p: X=\mathcal{C} \times \mathcal{M} S \rightarrow \mathcal{C}
$$


be the projection.

Definition 3.4. $\mathcal{E} n d(E)^{-1}:=\operatorname{res}^{-1}(\mathcal{E} n d(E)) \subset{ }^{\operatorname{tr}} \mathcal{A}_{E}^{-1}$, and

$$
\mathcal{G}_{E}:=\operatorname{res}^{-1}(\mathbb{E}) \subset \mathcal{E} n d(E)^{-1}
$$

where $\mathcal{E} n d(E)=\mathbb{E} \oplus \mathcal{O}_{X}$ with its trace free part $\mathbb{E}:=\mathcal{E} n d^{0}(E)$ and the trivial bundle $\mathcal{O}_{X}$. There are exact sequences

$$
\begin{gathered}
0 \rightarrow \omega_{X / S} \rightarrow \mathcal{G}_{E} \stackrel{\text { res }}{\longrightarrow} \mathbb{E} \rightarrow 0 ; \\
0 \rightarrow \omega_{X / S} \rightarrow \mathcal{E} n d(E)^{-1} \stackrel{\text { res }}{\longrightarrow} \mathcal{E} n d(E) \rightarrow 0 .
\end{gathered}
$$

Consider the natural morphism

$$
S^{2}\left(\mathcal{G}_{E}\right) \otimes_{\mathcal{O}_{X}} T_{X / S} \stackrel{q}{\rightarrow} S^{2}(\mathbb{E}) \otimes \mathcal{O}_{X} T_{X / S} \rightarrow 0
$$

induced by $\mathcal{G}_{E} \stackrel{\text { res }}{\longrightarrow} \mathbb{E}$. Denote the kernel of $q$ by $\mathcal{K}$. There is a canonical isomorphism $\iota: \mathcal{G}_{E} \cong \mathcal{K}$, that is $\iota(s)=\operatorname{Sym}^{2}(d t \otimes s) \otimes \partial_{t}$ locally.

Definition 3.5. $q^{-1}\left(\mathrm{id} \otimes T_{X / S}\right):=S\left(\mathcal{G}_{E}\right)$ where id $\in S^{2}(\mathbb{E})$ is the identity element. It follows that we have the exact sequence

$$
0 \rightarrow \mathcal{G}_{E} \stackrel{\iota}{\longrightarrow} S\left(\mathcal{G}_{E}\right) \rightarrow T_{X / S} \rightarrow 0
$$

Locally, for choosen coordinate and trivilization (cf. (3.7)), any local section $s \in S\left(\mathcal{G}_{E}\right)$ is of the form

$$
s=\left(\begin{array}{l}
\chi \sum_{a}\left(0, J_{a}, 0\right) \otimes\left(0, J_{a}, 0\right)+\sum_{a} \mu_{a}\left(0, J_{a}, 0\right) \otimes(0,0,1) \\
+\sum_{a} \nu_{a}(0,0,1) \otimes\left(0, J_{a}, 0\right)+w(0,0,1) \otimes(0,0,1)
\end{array}\right) \otimes \partial_{t}
$$

where $J_{a}$ is a (local) basis of $\mathbb{E}$ (which we assume to be orthonormal under Trace $(\cdot)$ ) such that $\sum_{a} J_{a} \otimes J_{a}=$ id (such a canonical choice of $J_{a}$ will simplify computations later).

We will need the Kodaira-Spencer maps

$$
\begin{gathered}
\mathrm{KS}_{S}: T_{S} \rightarrow R^{1} \pi_{*} \mathcal{A}_{E / S}^{0}, \quad \mathcal{A}_{E / S}^{0}=\mathcal{A}_{E / S} / \mathcal{O}_{X} ; \\
\mathrm{KS}_{\mathcal{M}}: f^{*} T_{\mathcal{M}} \rightarrow R^{1} \pi_{*} T_{X / S} .
\end{gathered}
$$

They fit into the following commutative diagram:

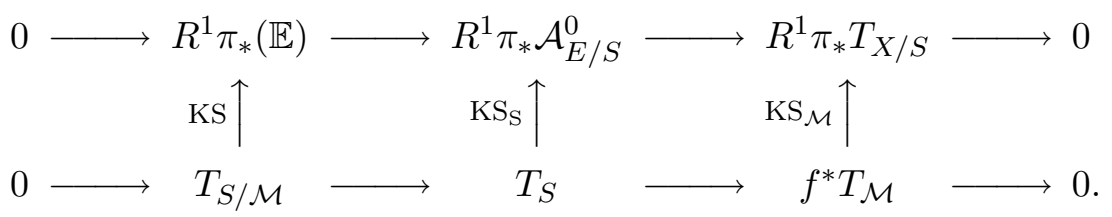


Remark 3.6. One way to see $\mathrm{KS}_{S}$ of (3.10) is via the natural map (with cohomology) $\mathcal{A}_{E, \pi}^{\bullet} \rightarrow \mathcal{A}_{E / S}^{0}[1]$ from (3.6); similarly $\mathrm{KS}_{\mathcal{M}}$ is via $\left(T_{\mathcal{C} / \mathcal{M}} \rightarrow T_{\pi}\right) \rightarrow T_{\mathcal{C} / \mathcal{M}}[1]$ (with cohomology) combined with its pullback via $f: S \rightarrow \mathcal{M}$. The diagram (3.10) via natural maps $T_{S} \rightarrow f^{*} T_{\mathcal{M}}$ and $R^{1} \pi_{*} \mathcal{A}_{E / S}^{0} \rightarrow R^{1} \pi_{*} T_{X / S}$, for Kodaira-Spencer maps commute.

\section{Theorem 3.7.}

i) If the Kodaira-Spencer map $\mathrm{KS}: T_{S / \mathcal{M}} \rightarrow R^{1} \pi_{*}(\mathbb{E})$ is an isomorphism, then there exists a canonical isomorphism

$$
\phi: R^{1} \pi_{*}\left(\mathcal{G}_{E}\right) \cong R^{0} \pi_{*}\left(\mathcal{E} n d(\mathbb{E})^{-1} \rightarrow \mathcal{A}_{\mathbb{E}, \pi / \mathcal{M}}\right) \cong R^{0} \pi_{*}{ }^{\operatorname{tr}} \mathcal{A}_{\mathbb{E} / \mathcal{M}}^{\bullet}
$$

ii) If $\mathrm{KS}_{S}$ is an isomorphism and shrink $\mathcal{M}$ enough, then the above $\phi$ extends to an isomorphism

$$
\phi=\phi_{-\lambda}: R^{1} \pi_{*} S\left(\mathcal{G}_{E}\right) \cong R^{0} \pi_{*}\left({ }^{\operatorname{tr}} \mathcal{A}_{\mathbb{E}}^{-1} \rightarrow \mathcal{A}_{\mathbb{E}, \pi}\right) .
$$

(The right-hand sides of i), ii) are canonically identified with $\mathcal{D}_{S / \mathcal{M}}^{\leq 1}\left(\lambda_{\mathbb{E}}\right)$, $\mathcal{D}_{S}^{\leq 1}\left(\lambda_{\mathbb{E}}\right)$ respectively, cf. Proposition 3.3.)

\section{Remark 3.8.}

$$
R^{0} \pi_{*}\left(\mathcal{E} n d(\mathbb{E})^{-1} \rightarrow \mathcal{A}_{\mathbb{E}, \pi / \mathcal{M}}\right) \cong R^{0} \pi_{*}\left(\mathcal{E} n d(\mathbb{E})^{-1} \rightarrow \mathcal{A}_{\mathbb{E}, \pi}\right)
$$

holds under $\mathcal{A}_{\mathbb{E}, \pi / \mathcal{M}} \rightarrow \mathcal{A}_{\mathbb{E}, \pi}$, cf. the proof of i) of Proposition 4.5.

Corollary 3.9. Assumptions being as in ii) of Theorem 3.7, suppose $\lambda_{\mathbb{E}} \cong \Theta^{-\lambda}(\lambda=2(r, d))$. For $k \in \mathbb{Z}$ one has an isomorphism denoted by

$$
\phi_{k}: R^{1} \pi_{*} S\left(\mathcal{G}_{E}\right) \cong \mathcal{D}_{S}^{\leq 1}\left(\Theta^{k}\right),
$$

extending Theorem 3.7. (If $k=-\lambda$, write $\phi$ for $\phi_{-\lambda}$.)

\section{Proposition 3.10.}

i) The morphism $\mathbb{E} \rightarrow \mathcal{E} n d^{0}(\mathbb{E})$ induced by the adjoint representation extends naturally to a canonical morphism ad : $\mathcal{A}_{E} \rightarrow \mathcal{A}_{\mathbb{E}}$ (preserving algebra structures).

ii) The morphism ad has a natural lifting $\widetilde{\mathrm{ad}}: \mathcal{G}_{E} \rightarrow \mathcal{G}_{\mathbb{E}}$, which induces $(2 r) \cdot$ id on $\omega_{X / S}$.

Proof.

i) For any $D \in \mathcal{A}_{E}, L \in \mathbb{E}, \operatorname{ad}(D)(L):=D \circ L-L \circ D$ is a section of $\mathbb{E}$ (note that $\operatorname{Tr}(D \circ L-L \circ D)=\epsilon_{1}(D) \operatorname{Tr}(L)$ ). Thus ad $(D)$ defines a map $\mathbb{E} \rightarrow \mathbb{E}$, which is a differential operator since $\operatorname{ad}(D)(\lambda \cdot L)=$ $\lambda \cdot \operatorname{ad}(D)(L)+\epsilon_{1}(D)(\lambda) \cdot L$. 
ii) This can be proved via the local formulas given in (3.7) Namely, a local element of $\mathcal{G}_{E}$ is expressed as $(0, B, \nu)$ (with $B \in \operatorname{Mat}_{r}\left(\mathcal{O}_{X}\right)$ and $\left.\nu \in \mathcal{O}_{X}\right)$. Define a lifting by sending $(0, B, \nu)$ to $(0, \operatorname{ad} B, 2 r \nu)$. We will show by using formulas in (3.8) that the above lifting is in fact globally defined. Using $\chi=0, \operatorname{Tr} B=0$ and $\operatorname{Tr}(\operatorname{ad} B)=0$ in (3.8), we have

$$
\begin{aligned}
& \quad(0, B, \nu) \stackrel{g}{\longrightarrow}\left(0, g B g^{-1}, \operatorname{Tr}\left(-B g^{-1} g^{\prime}\right)+\nu\right) \\
& (0, B, \nu) \stackrel{y(t)}{\longrightarrow}\left(0, B, \nu y^{\prime}\right) \\
& (0, \operatorname{ad} B, \nu) \stackrel{g}{\longrightarrow}\left(0, \operatorname{ad}\left(g B g^{-1}\right), \operatorname{Tr}\left(-\operatorname{ad} B \operatorname{ad}\left(g^{-1} g^{\prime}\right)\right)+\nu\right) ; \\
& (0, \operatorname{ad} B, \nu) \stackrel{y(t)}{\longrightarrow}\left(0, \operatorname{ad} B, \nu y^{\prime}\right) .
\end{aligned}
$$

If change the trivilization of $E$ by $g$, the induced trivilization of $\mathcal{E} n d(E)$ will be changed by $e_{g}: \mathbb{M}_{r}\left(\mathcal{O}_{X}\right) \rightarrow \mathbb{M}_{r}\left(\mathcal{O}_{X}\right)$, where $e_{g}(B)=g B g^{-1}$. It is easy to check that $e_{g}^{-1} e_{g}^{\prime}=\operatorname{ad}\left(g^{-1} g^{\prime}\right)$, thus we obtain the term $\operatorname{Tr}\left(-\operatorname{ad} B \operatorname{ad}\left(g^{-1} g^{\prime}\right)\right)$ in the 3rd row above. One knows that

$$
\operatorname{Tr}(\operatorname{ad} M \operatorname{ad} N)=2 r \operatorname{Tr}(M N)
$$

for traceless matrices $M, N$ of rank $r$. Let $\left(g^{-1} g^{\prime}\right)_{0}$ be the traceless compoment of $g^{-1} g^{\prime}$. Note $\operatorname{Tr} B=0, \operatorname{ad}\left(g^{-1} g^{\prime}\right)=\operatorname{ad}\left(\left(g^{-1} g^{\prime}\right)_{0}\right)$, and $\operatorname{Tr}\left(B g^{-1} g^{\prime}\right)=\operatorname{Tr}\left(B\left(g^{-1} g^{\prime}\right)_{0}\right)$ etc. It follows that the morphism $(0, B, \nu) \rightarrow(0, \operatorname{ad} B, 2 r \nu)$ as given is well-defined (globally). q.e.d.

Lemma 3.11. $R^{0} \pi_{*} \mathcal{A}_{E, \pi} \cong \mathcal{O}_{S}$ and $R^{0} \pi_{*} \mathcal{A}_{E, \pi}^{0}=0$ provided $\mathrm{KS}_{S}$ being injective, where $\mathcal{A}_{E, \pi}^{0}:=\mathcal{A}_{E, \pi} / \mathcal{O}_{X}$.

Proof. It suffices to prove, by using an exact sequence similar to (3.2) (with $T^{\bullet}$ replaced by $\mathcal{A}^{\bullet}$ ), that:

i) $\pi_{*} \pi^{-1} T_{S} \rightarrow R^{1} \pi_{*} \mathcal{A}_{E / S}$ is injective.

ii) $\pi_{*} \mathcal{A}_{E / S} \cong \mathcal{O}_{S}$.

But the map in i) composed with $R^{1} \pi_{*} \mathcal{A}_{E / S} \rightarrow R^{1} \pi_{*} \mathcal{A}_{E / S}^{0}$ is nothing but $\mathrm{KS}_{S}$, hence i). ii) is from $\pi_{*} T_{X / S}=0$ (genus $\geq 2$ ) and $\pi_{*} \mathcal{E} n d(E)=$ $\mathcal{O}_{S}(E$ is fiberwise stable). 
Proof of Theorem 3.7 i). First, we remark that morphisms in Proposition 3.10 make the following diagram of complexes commutative:

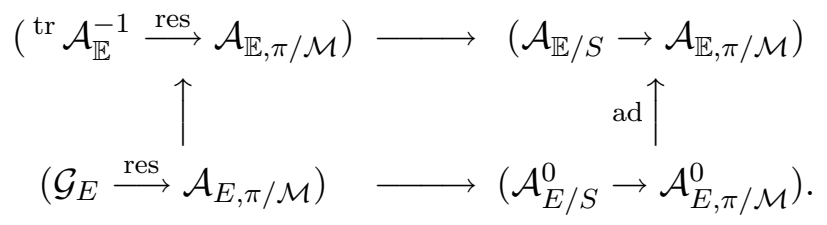

Second, we observe that the commutative diagram

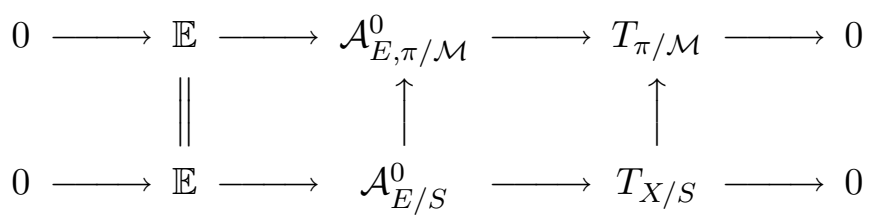

induces a commutative diagram

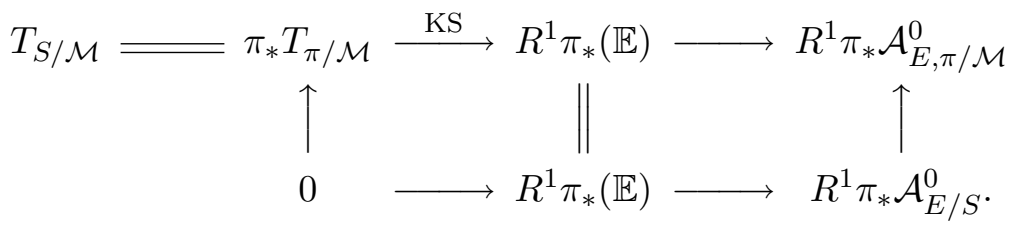

Thus $R^{1} \pi_{*}(\mathbb{E})$ vanishes in $R^{1} \pi_{*} \mathcal{A}_{E, \pi / \mathcal{M}}^{0}$ since $\mathrm{KS}$ is an isomorphism.

We construct $\phi$ for any affine open set $U^{i} \subset S$. Let $\left\{U_{i}, \dot{X}_{i}\right\}$ be an affine covering of $\pi^{-1}\left(U^{i}\right)$, let $\dot{U}_{i}=U_{i} \cap \dot{X}_{i}$. For any Čech cocycle $r_{\dot{U}_{i}} \in \mathcal{G}_{E}\left(\dot{U}_{i}\right)$ in $C^{1}\left(\mathcal{G}_{E}\right)$, the class $\left[\operatorname{res}\left(r_{\dot{U}_{i}}\right)\right] \in R^{1} \pi_{*}(\mathbb{E})\left(U^{i}\right)$ vanishes in $R^{1} \pi_{*} \mathcal{A}_{E, \pi / \mathcal{M}}^{0}\left(U^{i}\right)$. Thus there exists $\tau_{\dot{X}_{i}} \in \mathcal{A}_{E, \pi / \mathcal{M}}^{0}\left(\dot{X}_{i}\right), \tau_{U_{i}} \in$ $\mathcal{A}_{E, \pi / \mathcal{M}}^{0}\left(U_{i}\right)$ such that $\tau_{\dot{X}_{i}}-\tau_{U_{i}}=\operatorname{res} r_{\dot{U}_{i}}$ on $\dot{U}_{i}$. For given $r_{\dot{U}_{i}}$, the choice of $\tau_{\dot{X}_{i}}$ and $\tau_{U_{i}}$ is unique (by Lemma 3.11). Then

$$
\left\{\operatorname{ad}\left(\tau_{\dot{X}_{i}}\right), \operatorname{ad}\left(\tau_{U_{i}}\right) ; \widetilde{\operatorname{ad}}\left(r_{\dot{U}_{i}}\right)\right\}
$$

is a Čech cocycle in $C^{0}\left(\mathcal{G}_{\mathbb{E}} \rightarrow \mathcal{A}_{\mathbb{E}, \pi / \mathcal{M}}\right)($ cf. [2], p. 673). It is easily checked that the assignment

$$
\widetilde{\phi}: r_{\dot{U}_{i}} \rightarrow\left\{\operatorname{ad}\left(\tau_{\dot{X}_{i}}\right), \operatorname{ad}\left(\tau_{U_{i}}\right) ; \widetilde{\operatorname{ad}}\left(r_{\dot{U}_{i}}\right)\right\} \quad \in C^{0}\left({ }^{\operatorname{tr}} \mathcal{A}_{\mathbb{E} / \mathcal{M}}\right)
$$

preserves the respective coboundaries. Hence it descends to a map

$$
\phi: R^{1} \pi_{*}\left(\mathcal{G}_{E}\right) \rightarrow R^{0} \pi_{*}{ }^{\operatorname{tr}} \mathcal{A}_{\mathbb{E} / \mathcal{M}}^{\bullet}
$$


In the same way, we construct $\vartheta: R^{1} \pi_{*}(\mathbb{E}) \rightarrow R^{0} \pi_{*} \mathcal{A}_{\mathbb{E}, \pi / \mathcal{M}}^{\bullet}$ such that

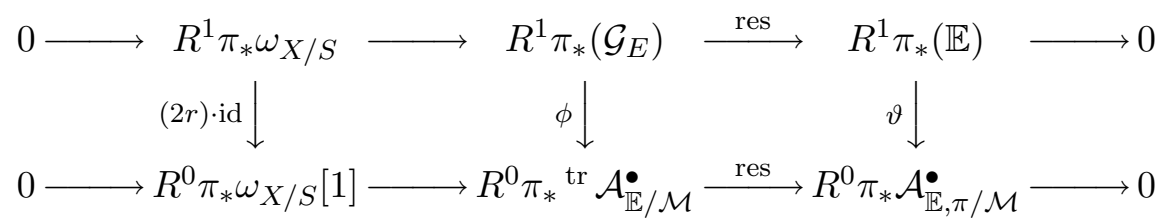

is commutative. The map $\vartheta$ is the composition of

$$
\mathrm{KS}^{-1}: R^{1} \pi_{*}(\mathbb{E}) \rightarrow T_{S / \mathcal{M}}=R^{0} \pi_{*}\left(\mathcal{A}_{E / S}^{0} \rightarrow \mathcal{A}_{E, \pi / \mathcal{M}}^{0}\right)
$$

and the map $R^{0} \pi_{*}\left(\mathcal{A}_{E / S}^{0} \rightarrow \mathcal{A}_{E, \pi / \mathcal{M}}^{0}\right) \rightarrow R^{0} \pi_{*} \mathcal{A}_{\mathbb{E}, \pi / \mathcal{M}}^{\bullet}$, which is induced by the qusi-isomorphism ad of complexes at the begining of our proof (it is a qusi-isomorphism since $\operatorname{ad}(D)$ has the same symbol with $D \in \mathcal{A}_{E}$ ). Thus $\vartheta$ is an isomorphism, then $\phi$ has to be an isomorphism. q.e.d.

Both $R^{1} \pi_{*}\left(\mathcal{G}_{E}\right)$ and $R^{0} \pi_{*}\left(\mathcal{E} n d(\mathbb{E})^{-1} \rightarrow \mathcal{A}_{\mathbb{E}, \pi / \mathcal{M}}\right)=R^{0} \pi_{*}{ }^{\operatorname{tr}} \mathcal{A}_{\mathbb{E} / \mathcal{M}}^{\bullet}$ define extension classes in $H^{1}\left(S, \Omega_{S / \mathcal{M}}\right)$. One has (by the preceding proof combined with proof of Proposition 3.10 for the constant $2 r$ ):

Corollary 3.12. The extension classes (e.c.[•] for short) satisfy

$$
\text { e.c. }\left[R^{1} \pi_{*}\left(\mathcal{G}_{E}\right)\right]=\frac{1}{2 r} \text { e.c. }\left[\mathcal{D}_{S}^{\leq 1} / \mathcal{M}\left(\lambda_{\mathbb{E}}\right)\right]
$$

in $H^{1}\left(S, \Omega_{S / \mathcal{M}}\right)$, where $\mathcal{D} \leq 1, \mathcal{M}\left(\lambda_{\mathbb{E}}\right)=R^{0} \pi_{*}\left(\mathcal{E} n d(\mathbb{E})^{-1} \rightarrow \mathcal{A}_{\mathbb{E}, \pi / \mathcal{M}}\right)$.

For ii) of the theorem, our proof will need the following result:

Proposition 3.13. There exisits $\widetilde{\text { res }}: S\left(\mathcal{G}_{E}\right) \rightarrow \mathcal{A}_{E / S}^{0}$ such that

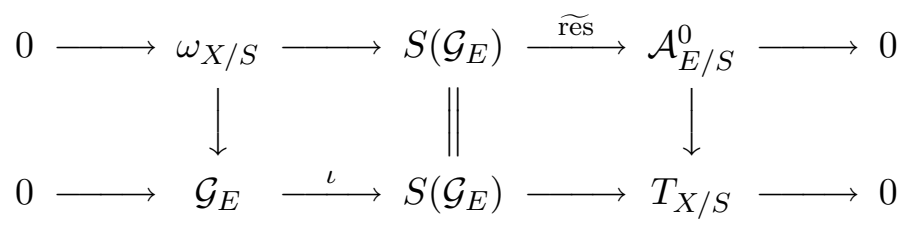

is commutative. If $\mathrm{KS}_{S}$ in (3.10) is an isomorphism, then

$$
0 \rightarrow \mathcal{O}_{S} \rightarrow R^{1} \pi_{*} S\left(\mathcal{G}_{E}\right) \rightarrow T_{S} \rightarrow 0 .
$$

Proof. For any local section $s \in S\left(\mathcal{G}_{E}\right)$ in Definition 3.5, we define

$$
\widetilde{\operatorname{res}}(s)=\left(\chi \partial_{t}, \frac{1}{2} \sum_{a}\left(\mu_{a}+\nu_{a}\right) J_{a}\right),
$$

which is independent of the choice of $\left\{J_{a}\right\}$, thus well-defined locally. To show it well-defined globally, we need to check the invariance under 
gauge and coordinate changes. The invariance under local coordinate changes is straightforward. Under the gauge change $g \in \mathrm{GL}_{r}\left(\mathcal{O}_{X}\right)$, the section $s \in S\left(\mathcal{G}_{E}\right)$ in Definition 3.5 becomes $s_{g} \otimes \partial_{t}$, where $s_{g}$ is

$$
\begin{aligned}
& \chi \sum_{a}\left(0, g J_{a} g^{-1}, 0\right)^{\otimes 2}+\sum_{a}\left(\mu_{a}-\chi \operatorname{Tr}\left(J_{a} g^{-1} g^{\prime}\right)\right)\left(0, g J_{a} g^{-1}, 0\right) \otimes(0,0,1) \\
& +\sum_{a}\left(\nu_{a}-\chi \operatorname{Tr}\left(J_{a} g^{-1} g^{\prime}\right)\right)(0,0,1) \otimes\left(0, g J_{a} g^{-1}, 0\right) \\
& +\left(\chi \sum_{a} \operatorname{Tr}\left(J_{a} g^{-1} g^{\prime}\right)^{2}-\sum_{a}\left(\mu_{a}+\nu_{a}\right) \operatorname{Tr}\left(J_{a} g^{-1} g^{\prime}\right)+w\right)(0,0,1)^{\otimes 2} .
\end{aligned}
$$

Then $\widetilde{\text { res }}\left(s_{g} \otimes \partial_{t}\right)=\left(\chi \partial_{t}, \frac{1}{2} \sum_{a}\left(\mu_{a}+\nu_{a}-2 \chi \operatorname{Tr}\left(J_{a} g^{-1} g^{\prime}\right)\right) g J_{a} g^{-1}\right)$ coincides with $\left(\chi \partial_{t},-\chi \partial_{t}(g) g^{-1}+\frac{1}{2} \sum_{a}\left(\mu_{a}+\nu_{a}\right) g J_{a} g^{-1}\right)$ in $\mathcal{A}_{E / S}^{0}=$ $\mathcal{A}_{E / S} / \mathcal{O}_{X}$ since $\left.\chi \partial_{t}(g) g^{-1}=\chi g^{\prime} g^{-1}=\sum_{a} \chi \operatorname{Tr}\left(J_{a} g^{-1} g^{\prime}\right)\right) g J_{a} g^{-1} \bmod -$ ulo $\mathcal{O}_{X}$. Thus res is gauge invariant and defined globally. Then the rest of this proposition is obvious.

q.e.d.

\section{Remark 3.14.}

i) $\operatorname{Sym}^{2}(a \otimes b)=\frac{1}{2}(a \otimes b+b \otimes a)$ for $a, b \in \mathcal{G}_{E}$.

ii) Using Proposition 3.13 and assuming $R^{1} f_{*} \mathcal{O}_{S}=0$ one has a quick interpretation of ii) of Theorem 3.7: note both $R^{1} \pi_{*} S\left(\mathcal{G}_{E}\right)$ and $R^{0} \pi_{*}{ }^{\operatorname{tr}} \mathcal{A}_{\mathbb{E}}^{\bullet} \cong \mathcal{D}_{\bar{S}}^{\leq 1}\left(\lambda_{\mathbb{E}}\right)$ contain a subsheaf $R^{1} \pi_{*}\left(\mathcal{G}_{E}\right) \cong \mathcal{D} \frac{\leq 1}{S / \mathcal{M}}\left(\lambda_{\mathbb{E}}\right)$. Given two extensions $\mathcal{F}, \mathcal{F}^{\prime}$ of $T_{S}$ by $\mathcal{O}_{S}$ suppose their subsheafs with symbolic part in $T_{S / \mathcal{M}}$ are isomorphic. Then $\mathcal{F} \cong \mathcal{F}^{\prime}$ (noncanonically) provided $R^{1} f_{*} \mathcal{O}_{S}=0$ (since

$$
H^{1}\left(S, \Omega_{S}\right) \rightarrow H^{1}\left(S, \Omega_{S / \mathcal{M}}\right)
$$

is injective if $R^{1} f_{*} \mathcal{O}_{S}=0$ (and $\mathcal{M}$ is affine)). Theorem 3.7 ii) just proves an isomorphism of this kind without reference to $R^{1} f_{*} \mathcal{O}_{S}=$ 0 .

In what follows we will choose an open covering of $\mathcal{C}$ (shrink $\mathcal{M}$ if necessary), fix a local coordinate (along the fiber) on each open set of the covering and use the local description (3.7). Recall that

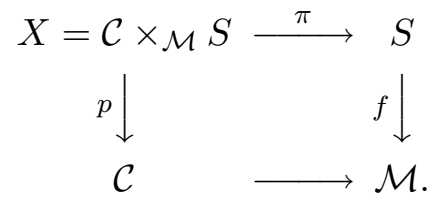


To simplify notation, we will cover $\mathcal{C}$ by two affine open sets $V$ and $\dot{\mathcal{C}}$. Let $V \subset \mathcal{C}$ be an affine open set and $t$ be a local coordinate (along the fiber) on $V$. Let $\dot{\mathcal{C}} \subset \mathcal{C}$ be an affine open set containing $\mathcal{C} \backslash V$. Then we choose and fix the local coordinates (along the fibre) on $V$ and $\dot{\mathcal{C}}$. Let $U=p^{-1}(V), \dot{X}=p^{-1}(\dot{\mathcal{C}})$ (they are not affine but affine over $S$ ). We start with the construction of $\gamma_{\mathbb{E}, U}:\left.\left.S\left(\mathcal{G}_{E}\right)\right|_{U} \rightarrow{ }^{\operatorname{tr}} \mathcal{A}_{\mathbb{E}}^{-1}\right|_{U}$ (resp. $\left.\gamma_{\mathbb{E}, \dot{X}}:\left.\left.S\left(\mathcal{G}_{E}\right)\right|_{\dot{X}} \rightarrow{ }^{\operatorname{tr}} \mathcal{A}_{\mathbb{E}}^{-1}\right|_{\dot{X}}\right)$ such that the following diagram is commutative over $U$ (resp. over $\dot{X})$ :

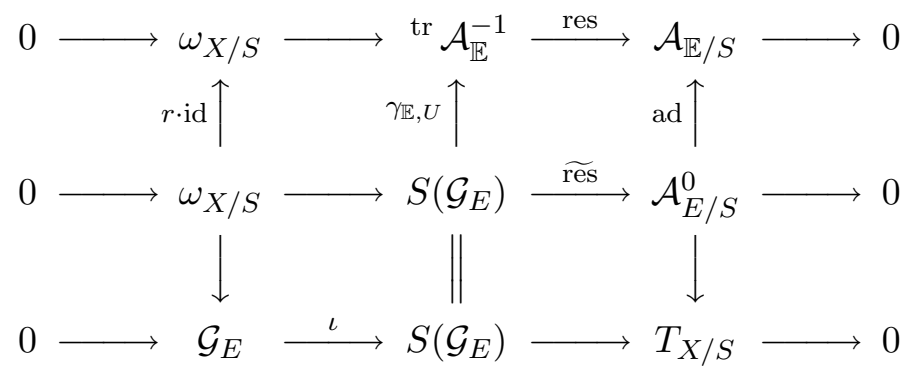

where ad $: \mathcal{A}_{E / S}^{0} \rightarrow \mathcal{A}_{\mathbb{E} / S}$ is induced by the morphism in Proposition $3.10 \mathrm{i})$ that maps $\mathcal{O}_{X}$ to zero. Note that, except $\gamma_{\mathbb{E}, U}\left(\operatorname{resp} . \gamma_{\mathbb{E}, \dot{X}}\right)$, other morphisms in the diagram are well-defined over the global $\mathcal{M}$ (i.e., need not shrink $\mathcal{M})$.

Let $\left\{U^{i}\right\}_{i}$ be an affine covering of $S$. Then $\left\{U_{i}:=U \cap \pi^{-1}\left(U^{i}\right)\right\}_{i}$ (resp. $\left.\left\{\dot{X}_{i}=\dot{X} \cap \pi^{-1}\left(U^{i}\right)\right\}_{i}\right)$ is an affine covering of $U$ (resp. $\left.\dot{X}\right)$. We will define $\gamma_{\mathbb{E}, U}\left(\right.$ resp. $\left.\gamma_{\mathbb{E}, \dot{X}}\right)$ on each $U_{i}\left(\right.$ resp. $\left.\dot{X}_{i}\right)$ using the local description (3.7), then prove it is defined globally on $U$ (resp. $\dot{X}$ ). It is important to remark that on each $U_{i}$ (resp. $\dot{X}_{i}$ ) we use the local coordinate pulling back from $V$ (resp. $\dot{\mathcal{C}}$ ). Thus, to prove it is well-defined on $U$ (resp. $\dot{X})$, we only need to check the independence of trivialization of $E$ over $U_{i}\left(\right.$ resp. $\left.\dot{X}_{i}\right)$. On each $U_{i}$, fix a trivilization $I$ of $E$ on $U_{i}$ and use the pullback coordinate (say, $t$ ) of $V$, we define the morphism by using the local description (3.7). For any local section $\alpha \in S\left(\mathcal{G}_{E}\right)\left(U_{i}\right)$, with respect to $I$ and $t$

$$
\alpha=\left(\begin{array}{l}
\chi \sum_{a}\left(0, J_{a}, 0\right) \otimes\left(0, J_{a}, 0\right)+\sum_{a} \mu_{a}\left(0, J_{a}, 0\right) \otimes(0,0,1) \\
+\sum_{a} \nu_{a}(0,0,1) \otimes\left(0, J_{a}, 0\right)+w(0,0,1) \otimes(0,0,1)
\end{array}\right) \otimes \partial_{t}
$$


(see the expression in Definition 3.5), one defines, where $r=\operatorname{rank}(E)$,

$$
\gamma_{\mathbb{E}, U}(\alpha)=\left(\chi, \frac{1}{2} \sum_{a}\left(\mu_{a}+\nu_{a}\right) \operatorname{ad} J_{a}, r w\right)_{(t, I)} \in{ }^{\operatorname{tr}} \mathcal{A}_{\mathbb{E}}^{-1}\left(U_{i}\right),
$$

where one uses the natural trivilization $I$ of $\mathbb{E}$ induced from that of $E$.

Lemma 3.15. The assignment $\alpha \rightarrow \gamma_{\mathbb{E}, U}(\alpha)$ constructed above is bundle gauge-invariant; More precisely:

i) Under another choice of trivialization of $E\left(\right.$ on $\left.U_{i}\right)$, such that $J_{a} \rightarrow$ $g J_{a} g^{-1}$ so that $\alpha \rightarrow \alpha_{g}$, the assignment to $\alpha_{g}$ becomes equal to the $e_{g}$-transformation of $\gamma_{\mathbb{E}, U}(\alpha)$, where $e_{g}$ is the gauge of $\mathbb{E}$ induced by $g$ (cf. Proposition 3.10).

ii) Under a coordinate change $y=y(t)$, the difference of $\gamma_{\mathbb{E}, U}(\alpha)$ is

$$
\left(0,0, r \chi\left(\frac{1}{6} \frac{y^{\prime \prime \prime}}{y^{\prime 2}}-\frac{1}{4} \frac{y^{\prime \prime 2}}{y^{\prime 3}}\right)\right)_{(t, I)} \in \omega_{X / S}(U) .
$$

If $\beta \in \mathcal{G}_{E}\left(U_{i}\right)$, then $\gamma_{\mathbb{E}, U}(\iota(\beta))=\frac{1}{2} \widetilde{\operatorname{ad}}(\beta)$, where $\widetilde{\text { ad }}: \mathcal{G}_{E} \rightarrow \mathcal{G}_{\mathbb{E}}$ is the morphism in ii) of Proposition 3.10.

Proof. The proof is to check a straightforward commutativity relation caused by the gauge change, hence we simplify the notation if there is no danger of confusion. As in the proof of Proposition 3.13, using the explicit expression of $\alpha_{g}=s_{g} \otimes \partial_{t}$, one has

$$
\begin{aligned}
\gamma_{\mathbb{E}, U}\left(\alpha_{g}\right)= & \left(\chi, \frac{1}{2} \sum_{a}\left(\mu_{a}+\nu_{a}-2 \chi \operatorname{Tr}\left(J_{a} g^{-1} g^{\prime}\right)\right) \operatorname{ad}\left(g J_{a} g^{-1}\right)\right. \\
& \left.r \chi \sum_{a} \operatorname{Tr}\left(J_{a} g^{-1} g^{\prime}\right)^{2}-r \sum_{a}\left(\mu_{a}+\nu_{a}\right) \operatorname{Tr}\left(J_{a} g^{-1} g^{\prime}\right)+r w\right) .
\end{aligned}
$$

The goal is to show $\gamma_{\mathbb{E}, U}\left(\alpha_{g}\right)$ equals the $e_{g}$-transformation $\gamma_{\mathbb{E}, U}(\alpha)^{g}$ of $\gamma_{\mathbb{E}, U}(\alpha)$, which is

$$
\left(\begin{array}{c}
\chi,-\chi e_{g}^{\prime} e_{g}^{-1}+\frac{1}{2} \sum_{a}\left(\mu_{a}+\nu_{a}\right) e_{g} \operatorname{ad}\left(J_{a}\right) e_{g}^{-1}, \quad r w+ \\
\operatorname{Tr}\left(-\frac{1}{2} \chi e_{g}^{\prime \prime} e_{g}^{-1}+\chi\left(e_{g}^{\prime} e_{g}^{-1}\right)^{2}-\frac{1}{2} \sum_{a}\left(\mu_{a}+\nu_{a}\right) \operatorname{ad}\left(J_{a}\right) e_{g}^{-1} e_{g}^{\prime}\right.
\end{array}\right)
$$

Recall that $e_{g}: \mathbb{M}_{r}\left(\mathcal{O}_{X}\right) \rightarrow \mathbb{M}_{r}\left(\mathcal{O}_{X}\right)$ means $e_{g}(B)=g B g^{-1}$, we have $e_{g} \operatorname{ad}\left(J_{a}\right) e_{g}^{-1}=\operatorname{ad}\left(g J_{a} g^{-1}\right)$. Thus the second components of $\gamma_{\mathbb{E}, U}\left(\alpha_{g}\right)$ and $\gamma_{\mathbb{E}, U}(\alpha)^{g}$ will coincide if $e_{g}^{\prime} e_{g}^{-1}=\sum_{a} \operatorname{Tr}\left(J_{a} g^{-1} g^{\prime}\right) \operatorname{ad}\left(g J_{a} g^{-1}\right)$, which 
is true since $e_{g}^{\prime} e_{g}^{-1}=\operatorname{ad}\left(g^{\prime} g^{-1}\right), e_{g}^{-1} e_{g}^{\prime}=\operatorname{ad}\left(g^{-1} g^{\prime}\right)$. To finish the proof, we will show that their third components coincide. Since

$$
\frac{1}{2} \sum_{a}\left(\mu_{a}+\nu_{a}\right) \operatorname{Tr}\left(\operatorname{ad}\left(J_{a}\right) e_{g}^{-1} e_{g}^{\prime}\right)=r \sum_{a}\left(\mu_{a}+\nu_{a}\right) \operatorname{Tr}\left(J_{a} g^{-1} g^{\prime}\right),
$$

it will be done if one can show the following identity:

$$
r \sum_{a} \operatorname{Tr}\left(J_{a} g^{-1} g^{\prime}\right)^{2}=\operatorname{Tr}\left(\left(e_{g}^{\prime} e_{g}^{-1}\right)^{2}-\frac{1}{2} e_{g}^{\prime \prime} e_{g}^{-1}\right) .
$$

Write $e_{g}^{\prime \prime} e_{g}^{-1}=\left(e_{g}^{\prime} e_{g}^{-1}\right)^{\prime}-e_{g}^{\prime}\left(e_{g}^{-1}\right)^{\prime}=\left(e_{g}^{\prime} e_{g}^{-1}\right)^{\prime}+e_{g}^{\prime} e_{g}^{-1} e_{g}^{\prime} e_{g}^{-1}$, then

$$
\operatorname{Tr}\left(e_{g}^{\prime \prime} e_{g}^{-1}\right)=\operatorname{Tr}\left(\left(e_{g}^{\prime} e_{g}^{-1}\right)^{2}\right)=\operatorname{Tr}\left(\operatorname{ad}\left(g^{\prime} g^{-1}\right) \operatorname{ad}\left(g^{\prime} g^{-1}\right)\right)
$$

(using $\left(e_{g} e_{g}^{-1}\right)^{\prime}=0$ and $\operatorname{Tr}\left(\left(e_{g}^{\prime} e_{g}^{-1}\right)^{\prime}\right)=\operatorname{Tr}\left(e_{g}^{\prime} e_{g}^{-1}\right)^{\prime}=0$ here). Let $\left(g^{\prime} g^{-1}\right)_{0}$ be the traceless part of $g^{\prime} g^{-1}$. Then R.H.S. of (3.16) equals

$$
\frac{1}{2} \operatorname{Tr}\left(\operatorname{ad}\left(g^{\prime} g^{-1}\right) \operatorname{ad}\left(g^{\prime} g^{-1}\right)\right)=r \operatorname{Tr}\left(\left(g^{\prime} g^{-1}\right)_{0}\left(g^{\prime} g^{-1}\right)_{0}\right) .
$$

By the choice of $\left\{J_{a}\right\}_{a}$, we have $\left(g^{-1} g^{\prime}\right)_{0}=\sum_{a} \operatorname{Tr}\left(J_{a} g^{-1} g^{\prime}\right) J_{a}$. Then L.H.S of (3.16) equals $r \operatorname{Tr}\left(\left(g^{-1} g^{\prime}\right)_{0} g^{-1} g^{\prime}\right)=r \operatorname{Tr}\left(\left(g^{-1} g^{\prime}\right)_{0}\left(g^{-1} g^{\prime}\right)_{0}\right)$. Thus (3.16) is true since $g\left(g^{-1} g^{\prime}\right)_{0} g^{-1}=\left(g^{\prime} g^{-1}\right)_{0}$. We are done with i). The assertion ii) is a straightforward calculation using the coordinate change formula (3.8) and $\partial_{y}=y^{\prime}(t)^{-1} \partial_{t}$. For $\beta=\left(0, \sum_{a} \xi_{a} J_{a}, w\right) \in \mathcal{G}_{E}\left(U_{i}\right)$, $\iota(\beta)=\frac{1}{2}\left(\left(0, \sum_{a} \xi_{a} J_{a}, w\right) \otimes(0,0,1)+(0,0,1) \otimes\left(0, \sum_{a} \xi_{a} J_{a}, w\right)\right) \otimes \partial_{t}$

$$
=\left(\begin{array}{l}
\sum_{a} \frac{1}{2} \xi_{a}\left(0, J_{a}, 0\right) \otimes(0,0,1)+\sum_{a} \frac{1}{2} \xi_{a}(0,0,1) \otimes\left(0, J_{a}, 0\right) \\
+w(0,0,1) \otimes(0,0,1)
\end{array}\right) \otimes \partial_{t} .
$$

Thus $\gamma_{\mathbb{E}, U}(\iota(\beta))=\frac{1}{2}\left(0, \operatorname{ad}\left(\sum_{a} \xi_{a} J_{a}\right), 2 r w\right)=\frac{1}{2} \widetilde{\operatorname{ad}}(\beta) . \quad$ q.e.d.

We have constructed $\gamma_{\mathbb{E}, U}$ (the construction of $\gamma_{\mathbb{E}, \dot{X}}$ is similar) such that the above diagram $(*)$ is commutative over $U$ (resp. over $\dot{X})$. However, on $\dot{U}:=U \cap \dot{X}$, the difference (caused by the difference of coordinates on $U$ and $\dot{X}), \gamma_{\dot{U}}:=\gamma_{\mathbb{E}, U}-\gamma_{\mathbb{E}, \dot{X}}$ vanishes on $\left.\mathcal{G}_{E}\right|_{\dot{U}}$, but may not vanish on $\left.S\left(\mathcal{G}_{E}\right)\right|_{\dot{U}}$ (cf. ii) of Lemma 3.15). It defines a morphism $\gamma_{\dot{U}}:\left.\left.S\left(\mathcal{G}_{E}\right)\right|_{\dot{U}} \rightarrow \omega_{X / S}\right|_{\dot{U}}$ that induces $\widetilde{\gamma}_{\dot{U}}:\left.\left.T_{X / S}\right|_{\dot{U}} \rightarrow \omega_{X / S}\right|_{\dot{U}}$, i.e., a section $\widetilde{\gamma}_{\dot{U}} \in \mathcal{H o m}\left(T_{X / S}, \omega_{X / S}\right)(\dot{U})=\omega_{X / S}^{2}\left(p^{-1}(\dot{V})\right)$, where $\dot{V}:=V \cap \dot{\mathcal{C}}$. The $\left[\widetilde{\gamma}_{\dot{U}}\right]$ defines a class of $H^{1}\left(\mathcal{C}, p_{*} \omega_{X / S}^{2}\right)=H^{1}\left(\mathcal{C}, \omega_{\mathcal{C} / \mathcal{M}}^{2}\right)$, which vanishes when $\mathcal{M}$ is replaced by an affine open set. Thus there exist

$$
\widetilde{\psi}_{U} \in \mathcal{H o m}\left(T_{X / S}, \omega_{X / S}\right)(U), \quad \widetilde{\psi}_{\dot{X}} \in \mathcal{H o m}\left(T_{X / S}, \omega_{X / S}\right)(\dot{X})
$$


such that $\widetilde{\gamma}_{\dot{U}}=\widetilde{\psi}_{U}-\widetilde{\psi}_{\dot{X}}$. Let $\psi_{U}, \psi_{\dot{X}}$ denote the induced morphisms

$$
\begin{gathered}
\psi_{U}:\left.S\left(\mathcal{G}_{E}\right)\right|_{U} \rightarrow S\left(\mathcal{G}_{E}\right) /\left.\left.\left.\mathcal{G}_{E}\right|_{U} \cong T_{X / S}\right|_{U} \stackrel{\widetilde{\psi}_{U}}{\longrightarrow} \omega_{X / S}\right|_{U} \\
\psi_{\dot{X}}:\left.S\left(\mathcal{G}_{E}\right)\right|_{\dot{X}} \rightarrow S\left(\mathcal{G}_{E}\right) /\left.\left.\left.\mathcal{G}_{E}\right|_{\dot{X}} \cong T_{X / S}\right|_{\dot{X}} \stackrel{\widetilde{\psi}_{\dot{X}}}{\longrightarrow} \omega_{X / S}\right|_{\dot{X}} \cdot
\end{gathered}
$$

Then it is easy to see that $\gamma_{\dot{U}}=\gamma_{\mathbb{E}, U}-\gamma_{\mathbb{E}, \dot{X}}=\psi_{U}-\psi_{\dot{X}}$. Let

$$
\beta_{U}:=\gamma_{\mathbb{E}, U}-\psi_{U}, \quad \beta_{\dot{X}}:=\gamma_{\mathbb{E}, \dot{X}}-\psi_{\dot{X}}
$$

Thus, by shrinking $\mathcal{M}$, we have proved:

Proposition 3.16. The $\beta_{U}$ and $\beta_{\dot{X}}$ define a morphism

$$
\beta: S\left(\mathcal{G}_{E}\right) \rightarrow{ }^{\operatorname{tr}} \mathcal{A}_{\mathbb{E}}^{-1},
$$

which induces (through ८) $\frac{1}{2} \widetilde{\mathrm{ad}}$ on $\mathcal{G}_{E}$, such that the following diagram is commutative:

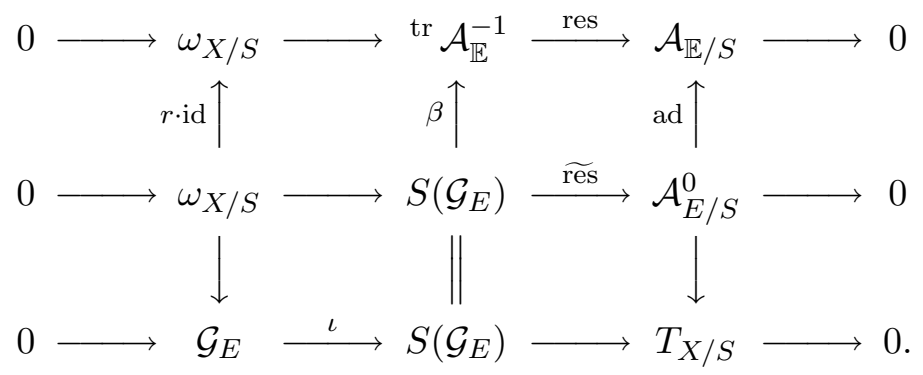

Proof of Theorem 3.7 ii). The proof is similar to that of i) of the theorem. By

$$
0 \rightarrow \mathcal{A}_{E / S}^{0} \rightarrow \mathcal{A}_{E, \pi}^{0} \rightarrow \pi^{-1} T_{S} \rightarrow 0
$$

and by identifying its connecting map $T_{S} \rightarrow R^{1} \pi_{*} \mathcal{A}_{E / S}^{0}$ with the Kodaira-Spencer map $\mathrm{KS}_{S}$ (which will be treated more generally for $\log D$ in Proposition 4.5), we see that $R^{1} \pi_{*} \mathcal{A}_{E / S}^{0}$ vanishes in $R^{1} \pi_{*} \mathcal{A}_{E, \pi}^{0}$ if $\mathrm{KS}_{S}$ is an isomorphism. Thus, for any $\alpha \in S\left(\mathcal{G}_{E}\right)\left(\dot{U}_{i}\right)$, there exist $\tau_{\dot{X}_{i}} \in \mathcal{A}_{E, \pi}^{0}\left(\dot{X}_{i}\right)$ and $\tau_{U_{i}} \in \mathcal{A}_{E, \pi}^{0}\left(U_{i}\right)$ such that $\tau_{\dot{X}_{i}}-\tau_{U_{i}}=\widetilde{\operatorname{res}}(\alpha)$. Then, by Proposition 3.16, we see that $\operatorname{res}(\beta(\alpha))=\operatorname{ad}(\widetilde{\operatorname{res}}(\alpha))$. Thus

$$
\widetilde{\phi}(\alpha):=\left\{\operatorname{ad}\left(\tau_{\dot{X}_{i}}\right), \operatorname{ad}\left(\tau_{U_{i}}\right) ; \beta(\alpha)\right\}
$$

is a cocycle in $C^{0}\left({ }^{\operatorname{tr}} \mathcal{A}_{\mathbb{E}}^{-1} \rightarrow \mathcal{A}_{\mathbb{E}, \pi}\right)$. It is clear that $\widetilde{\phi}$ induces a morphism

$$
\phi: R^{1} \pi_{*} S\left(\mathcal{G}_{E}\right) \rightarrow R^{0} \pi_{*}\left({ }^{\operatorname{tr}} \mathcal{A}_{\mathbb{E}}^{-1} \rightarrow \mathcal{A}_{\mathbb{E}, \pi}\right)=R^{0} \pi_{*}{ }^{\operatorname{tr}} \mathcal{A}_{\mathbb{E}}^{\bullet} .
$$


Similarly, we construct $\vartheta: R^{1} \pi_{*} \mathcal{A}_{E / S}^{0} \rightarrow R^{0} \pi_{*} \mathcal{A}_{\mathbb{E}, \pi}^{\bullet}$, which is the composition of

$$
\mathrm{KS}_{S}^{-1}: R^{1} \pi_{*} \mathcal{A}_{E / S}^{0} \rightarrow T_{S}=R^{0} \pi_{*}\left(\mathcal{A}_{E / S}^{0} \rightarrow \mathcal{A}_{E, \pi}^{0}\right)
$$

and the isomorphism $R^{0} \pi_{*}\left(\mathcal{A}_{E / S}^{0} \rightarrow \mathcal{A}_{E, \pi}^{0}\right) \rightarrow R^{0} \pi_{*} \mathcal{A}_{\mathbb{E}, \pi}^{\bullet}$ (induced by ad). The $\vartheta$ is an isomorphism such that the following diagram:

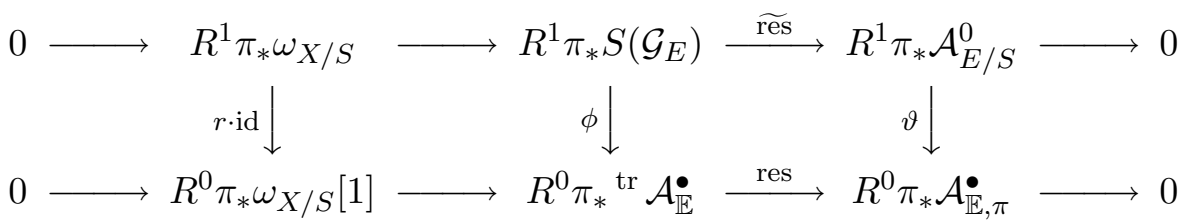

is commutaive. Thus $\phi$ must be an isomorphism.

q.e.d.

Remark 3.17. The $\phi$ in i) of Theorem 3.7 is defined globally over $\mathcal{M}$, but the one in ii) of Theorem 3.7 is defined only over an open set of $\mathcal{M}$. More precisely, there is an affine covering $\left\{\mathcal{M}_{i}\right\}_{i \in I}$ of $\mathcal{M}$ such that on each $\mathcal{C} \times{ }_{\mathcal{M}} f^{-1}\left(\mathcal{M}_{i}\right)$ we can choose a $\beta_{i}: S\left(\mathcal{G}_{E}\right) \rightarrow{ }^{\text {tr }} \mathcal{A}_{\mathbb{E}}^{-1}$ as the $\beta$ in Proposition 3.16. Then, by using $\beta_{i}$ and ii) of Theorem 3.7, we get the isomorphism $\phi_{i}: R^{1} \pi_{*} S\left(\mathcal{G}_{E}\right) \rightarrow R^{0} \pi_{*}{ }^{\text {tr }} \mathcal{A}_{\mathbb{E}}^{\bullet}=\mathcal{D}_{S}^{\leq 1}\left(\lambda_{\mathbb{E}}\right)$ on $f^{-1}\left(\mathcal{M}_{i}\right)$. For another choice $\left\{\beta_{i}^{\prime}\right\}_{i \in I}$, the map $\beta_{i}-\beta_{i}^{\prime}: S\left(\mathcal{G}_{E}\right) \rightarrow \omega_{X / S}$ induces

$$
\phi_{i}-\phi_{i}^{\prime}: R^{1} \pi_{*} S\left(\mathcal{G}_{E}\right) \rightarrow R^{0} \pi_{*} \omega_{X / S}[1]=\mathcal{O}_{S}
$$

on $f^{-1}\left(\mathcal{M}_{i}\right)$, which vanishes on $R^{1} \pi_{*}\left(\mathcal{G}_{E}\right)$ (cf. Theorem $3.7 \mathrm{i}$, which is global on $\mathcal{M})$, thus $\left(\phi_{i}-\phi_{i}^{\prime}\right) \in \Omega_{\mathcal{M}}^{1}\left(\mathcal{M}_{i}\right)$. Similarly, on $f^{-1}\left(\mathcal{M}_{i} \cap \mathcal{M}_{j}\right)$, $\phi_{i j}:=\phi_{i}-\phi_{j}$ induces $\widetilde{\phi}_{i j} \in \Omega_{\mathcal{M}}^{1}\left(\mathcal{M}_{i} \cap \mathcal{M}_{j}\right)$. Thus $\left\{\widetilde{\phi}_{i j}\right\}$ defines a class in $\mathrm{H}^{1}\left(\mathcal{M}, \Omega_{\mathcal{M}}^{1}\right)$, which is the obstruction of extending the isomorphism in ii) of Theorem 3.7 to the global $\mathcal{M}$.

To conclude this section, we describe the connecting maps $\widetilde{\delta}$ and prove Lemma 2.4 (cf. Lemma 3.18).

Lemma 3.18. The map $\widetilde{\delta}$ induces the identity map on $R^{1} \pi_{*}\left(\mathcal{G}_{E}\right)$. More precisely, $\widetilde{\delta} \circ \iota(\bullet)=\operatorname{Sym}^{2}((\bullet) \otimes 1)$.

Proof. It is known (see Proposition 4.2 of [16]) that the connecting map

$$
\widetilde{\delta}: R^{1} \pi_{*}\left(\left.\mathcal{G}_{E} \otimes \mathcal{G}_{E}(\Delta)\right|_{\Delta}\right) \rightarrow R^{2}(\pi \times \pi)_{*}\left(\mathcal{G}_{E} \otimes \mathcal{G}_{E}\right)
$$

is dual (under Serre duality) to the restriction map

$$
r:(\pi \times \pi)_{*}\left(\mathcal{G}_{E}^{*} \otimes \mathcal{G}_{E}^{*} \otimes \omega_{X / S} \otimes \omega_{X / S}\right) \rightarrow \pi_{*}\left(\mathcal{G}_{E}^{*} \otimes \mathcal{G}_{E}^{*} \otimes \omega_{\Delta / S}^{2}\right) .
$$


Working locally on $S$, we can assume that $X$ is covered by affine open sets $U$ and $\dot{X}$. Let $w \in \omega_{X / S}(\dot{U})$ be a base of $\omega_{X / S}$ on $\dot{U}:=U \cap \dot{X}$ and $w^{*} \in T_{X / S}(\dot{U})$ be its dual base. Then, for any $\alpha \in \mathcal{G}_{E}(\dot{U})$,

$$
\iota([\alpha])=\left[\operatorname{Sym}^{2}(\alpha \otimes w) \otimes w^{*}\right]=\frac{1}{2}\left[(\alpha \otimes w+w \otimes \alpha) \otimes w^{*}\right] .
$$

We use the following identification:

$(\pi \times \pi)_{*}\left(\mathcal{G}_{E}^{*} \otimes \mathcal{G}_{E}^{*} \otimes \omega_{X / S} \otimes \omega_{X / S}\right) \cong \pi_{*}\left(\mathcal{G}_{E}^{*} \otimes \omega_{X / S}\right) \otimes \pi_{*}\left(\mathcal{G}_{E}^{*} \otimes \omega_{X / S}\right)$.

For any $\beta_{i} \in \pi_{*}\left(\mathcal{G}_{E}^{*} \otimes \omega_{X / S}\right)(i=1,2)$, let $\left.\beta_{i}\right|_{\dot{U}}=s_{i} \otimes w$, where $s_{i} \in$ $\mathcal{G}_{E}^{*}(\dot{U})(i=1,2)$. Then $\left.r\left(\beta_{1} \otimes \beta_{2}\right)\right|_{\dot{U}}=s_{1} \otimes s_{2} \otimes w \otimes w$. Thus

$$
\left\langle\widetilde{\delta}(\iota([\alpha])), \beta_{1} \otimes \beta_{2}\right\rangle=\frac{1}{2}\left[\left(s_{1}(\alpha) s_{2}(w)+s_{1}(w) s_{2}(\alpha)\right) \cdot w\right] .
$$

By $R^{1} \pi_{*} \omega_{X / S}=\mathcal{O}_{S}$, let $[f \cdot w]=1 \in \mathcal{O}_{S}\left(f \in \mathcal{O}_{X}(\dot{U})\right)$, we have

$$
\begin{aligned}
& \left\langle\operatorname{Sym}^{2}([\alpha] \otimes[f \cdot w]), \beta_{1} \otimes \beta_{2}\right\rangle \\
& =\frac{1}{2}\left(\left[s_{1}(\alpha) \cdot w\right]\left[f s_{2}(w) \cdot w\right]+\left[f s_{1}(w) \cdot w\right]\left[s_{2}(\alpha) \cdot w\right]\right) .
\end{aligned}
$$

Note that $s_{i}(w)=\left.\beta_{i}\right|_{\dot{U}}\left(w \otimes w^{*}\right)(i=1,2)$, we can see that $s_{i}(w)=$ $\beta_{i}$ (id) $\left.\right|_{\dot{U}}$, where the (global) section id is the image of 1 under $\mathcal{O}_{X} \rightarrow$ $\mathcal{G}_{E} \otimes \omega_{X / S}^{*}$. Thus $s_{i}(w) \in \mathcal{O}_{S}$ since $\beta_{i}(i=1,2)$ are global sections of $\left(\mathcal{G}_{E} \otimes \omega_{X / S}^{*}\right)^{*}$. Then $\left[s_{1}(\alpha) s_{2}(w) \cdot w\right]=\left[s_{1}(\alpha) \cdot w\right]\left[f s_{2}(w) \cdot w\right]$ and $\left[s_{2}(\alpha) s_{1}(w) \cdot w\right]=\left[s_{2}(\alpha) \cdot w\right]\left[f s_{1}(w) \cdot w\right]$, which means that

$$
\langle\widetilde{\delta}(\iota([\alpha])), \bullet\rangle=\left\langle\operatorname{Sym}^{2}([\alpha] \otimes 1), \bullet\right\rangle .
$$

q.e.d.

\section{Generalization to the singular case}

Let $\pi: \widetilde{X} \rightarrow \widetilde{S}$ be a proper morphism of relative dimension 1 between smooth varieties in characteristic 0 such that each fiber has at most ordinary double points as singularities. Let $\widetilde{f}: \widetilde{S} \rightarrow \widetilde{\mathcal{M}}$ be a smooth morphism where $\widetilde{\mathcal{M}}$ is a smooth variety. Let $B=\widetilde{\mathcal{M}} \backslash \mathcal{M}$ and $W=$ $\tilde{f}^{-1}(B)$ such that $D=\pi^{-1}(W)$ consists precisely of singular fibres. As before let $\omega_{\tilde{X} / \widetilde{S}}$ be the relative dualizing sheaf (which is locally free as is well-known). Let $T_{\widetilde{\mathcal{M}}}(B) \subset T_{\widetilde{\mathcal{M}}}$ be the subalgebra of vector fields that preserve $B$ (cf. [2], Section 6 ). 
Notation 4.1. In the notation of Section 3, define the following:

$$
\begin{aligned}
T_{\widetilde{f}}(\log D) & =d(\widetilde{f} \circ \pi)^{-1}\left((\widetilde{f} \circ \pi)^{-1} T_{\widetilde{\mathcal{M}}}(B)\right) \subset T_{\widetilde{X}} ; \\
T_{\pi}(\log D) & =T_{\widetilde{f}}(\log D) \cap d \pi^{-1}\left(\pi^{-1} T_{\widetilde{S}}\right) \subset T_{\widetilde{X}} ; \\
T_{\widetilde{S}}(\log W) & =d \pi\left(T_{\pi}(\log D)\right) \subset T_{\widetilde{S}} ; \\
T_{\widetilde{X} / \widetilde{S}}(\log D) & =T_{\widetilde{X} / \widetilde{S}} \cap T_{\pi}(\log D) .
\end{aligned}
$$

Notation 4.2. Let $E$ be a vector bundle on $\widetilde{X}$. Define the following:

$$
\begin{aligned}
& \mathcal{A}_{E, \pi}(\log D)=\epsilon^{-1} T_{\pi}(\log D) \subset \mathcal{A}_{E} ; \\
& \mathcal{A}_{E / \widetilde{S}}(\log D)=\epsilon^{-1} T_{\widetilde{X} / \widetilde{S}}(\log D) \subset \mathcal{A}_{E} ; \\
& \mathcal{A}_{\lambda_{E}}(\log W)=\epsilon^{-1} T_{\widetilde{S}}(\log W) \subset \mathcal{A}_{\lambda_{E}},
\end{aligned}
$$

where the " $\epsilon$ "s denote symbol maps.

The ${ }^{\operatorname{tr}} \mathcal{A}_{E}^{-1}$ in Section 3 admits a generalization ${ }^{\operatorname{tr}} \mathcal{A}_{E}^{-1}(\log D)$ (cf. [19], p. 593) such that

$$
0 \rightarrow \omega_{\tilde{X} / \widetilde{S}} \rightarrow{ }^{\operatorname{tr}} \mathcal{A}_{E}^{-1}(\log D) \rightarrow \mathcal{A}_{E / \widetilde{S}}(\log D) \rightarrow 0,
$$

is exact. Furthermore with ${ }^{\operatorname{tr}} \mathcal{A}_{E}^{\bullet}$ in Section 3 replaced by ${ }^{\operatorname{tr}} \mathcal{A}_{E}^{\bullet}(\log D)$ (with $\mathcal{A}_{E, \pi}(\log D)$ in degree 0 ), one has:

Proposition 4.3 (cf. [19]). There is a canonical isomorphism

$$
R^{0} \pi_{*}^{\operatorname{tr}} \mathcal{A}_{E}^{\bullet}(\log D) \cong \mathcal{A}_{\lambda_{E}}(\log W)
$$

that extends Proposition 3.1.

Now go back to moduli situation, note $T_{\widetilde{X} / \widetilde{S}}(\log D)=\omega_{\widetilde{X} / \widetilde{S}}^{-1}$. Recall Notation 2.10 and Kodaira-Spencer map (cf. [20], Remark 3.2.7)

$$
\mathrm{KS}: \widetilde{f}^{*} T_{\widetilde{\mathcal{M}}}(B) \rightarrow R^{1} \pi_{*} T_{\widetilde{X} / \widetilde{S}}(\log D)
$$

As in the situation of smooth curves, we have:

Lemma 4.4. The morphism $\widetilde{f}: \widetilde{S} \rightarrow \widetilde{\mathcal{M}}$ is smooth and

$$
T_{\widetilde{S} / \widetilde{M}}=R^{1} \pi_{*} \mathcal{E} n d^{0}(E) .
$$

Proof. When $C$ is irreducible, its fibre $\widetilde{f}^{-1}([C])$ is the moduli space of stable bundles with fixed determinant $\left.\mathcal{N}\right|_{C}$. When $C$ is reducible, $\tilde{f}^{-1}([C])$ have a few disjoint irreducible components and each component consists of bundles with a fixed determinant that coincides with $\left.\mathcal{N}\right|_{C}$ outside the node of $C(\mathrm{cf} .[\mathbf{1 8}])$.

q.e.d. 


\section{Proposition 4.5.}

i) Assume the Kodaira-Spencer map KS (4.2) is injective. Then $R^{0} \pi_{*}\left(\mathcal{E} n d(E)^{-1} \rightarrow \mathcal{A}_{E, \pi}(\log D)\right) \rightarrow R^{0} \pi_{*}\left(\mathcal{E} n d(E) \rightarrow \mathcal{A}_{E, \pi}(\log D)\right) \rightarrow 0$ is canonically isomorphic to

$$
\mathcal{D} \underset{\widetilde{S} / \widetilde{\mathcal{M}}}{\leq 1}\left(\lambda_{E}\right) \rightarrow T_{\widetilde{S} / \widetilde{\mathcal{M}}} \rightarrow 0
$$

ii) Assume that $\mathrm{KS}(4.2)$ is an isomorphism. Then

$$
R^{1} \pi_{*} \mathcal{A}_{E / \widetilde{S}}^{0}(\log D) \cong T_{\widetilde{S}}(\log W)
$$

canonically, where $\mathcal{A}_{E / \widetilde{S}}^{0}(\log D)=\mathcal{A}_{E / \widetilde{S}}(\log D) / \mathcal{O}_{\tilde{X}}$.

The following is left to the reader:

\section{Lemma 4.6.}

$$
\begin{aligned}
\left(\mathcal{A}_{E / \widetilde{S}}(\log D) \rightarrow \mathcal{A}_{E, \pi}(\log D)\right) & \cong_{\mathrm{q} . \mathrm{i} .}\left(T_{\widetilde{X} / \widetilde{S}}(\log D) \rightarrow T_{\pi}(\log D)\right) \\
& \cong_{\mathrm{q} . \mathrm{i} .} \pi^{-1} T_{\widetilde{S}}(\log W)
\end{aligned}
$$

as quasi-isomorphisms.

Proof of Proposition 4.5.

i) From the exact sequence

$0 \rightarrow\left(\mathcal{E} n d(E)^{-1} \rightarrow \mathcal{A}_{E, \pi}(\log D)\right) \rightarrow^{\operatorname{tr}} \mathcal{A}_{E}^{\bullet}(\log D) \rightarrow T_{\widetilde{X} / \widetilde{S}}(\log D)[1] \rightarrow 0$, and passage to cohomology

$$
\begin{gathered}
0 \rightarrow R^{0} \pi_{*}\left(\mathcal{E} n d(E)^{-1} \rightarrow \mathcal{A}_{E, \pi}(\log D)\right) \rightarrow \mathcal{A}_{\lambda_{E}}(\log W) \stackrel{\epsilon}{\rightarrow} \\
R^{0} \pi_{*} T_{\widetilde{X} / \widetilde{S}}(\log D)[1] \rightarrow \cdots,
\end{gathered}
$$

one has, via the injectivity of KS, that $\epsilon^{-1}(0)$ has symbolic part in $T_{\widetilde{S} / \widetilde{\mathcal{M}}}$. This gives one of the isomorphisms in i) (the one with $D_{\widetilde{S} / \widetilde{\mathcal{M}}}^{\leq 1}\left(\lambda_{E}\right)$ ). Further, by

$$
\begin{aligned}
0 \rightarrow \omega_{\tilde{X} / \widetilde{S}}[1] \rightarrow\left(\mathcal{E} n d(E)^{-1}\right. & \left.\rightarrow \mathcal{A}_{E, \pi}(\log D)\right) \\
& \rightarrow\left(\mathcal{E} n d(E) \rightarrow \mathcal{A}_{E, \pi}(\log D)\right) \rightarrow 0
\end{aligned}
$$

and $R^{0} \pi_{*} \omega_{\widetilde{X} / \widetilde{S}}[1] \cong \mathcal{O}_{\widetilde{S}}$ it follows

$$
R^{0} \pi_{*}\left(\mathcal{E} n d(E)^{-1} \rightarrow \mathcal{A}_{E, \pi}(\log D)\right) \rightarrow R^{0} \pi_{*}\left(\mathcal{E} n d(E) \rightarrow \mathcal{A}_{E, \pi}(\log D)\right)
$$

is nothing but the symbol map, completing the asserted isomorphisms. 
ii) Write $B^{\bullet}(\log D)$ for $\left(\mathcal{E} n d(E) \rightarrow \mathcal{A}_{E, \pi}(\log D)\right)$ and $\mathcal{A}_{E, \pi}^{\bullet}(\log D)$ for L.H.S. of Lemma 4.6. The following exact sequence:

$$
0 \rightarrow B^{\bullet}(\log D) \rightarrow \mathcal{A}_{E, \pi}^{\bullet}(\log D) \rightarrow T_{\widetilde{X} / \widetilde{S}}(\log D)[1] \rightarrow 0
$$

projects to

$$
0 \rightarrow \mathcal{E} n d^{0}(E)[1] \rightarrow \mathcal{A}_{E / \widetilde{S}}^{0}(\log D)[1] \rightarrow T_{\widetilde{X} / \widetilde{S}}(\log D)[1] \rightarrow 0 .
$$

Computing direct images of (4.3) and (4.4), by i) just proved and Lemma 4.6, yields that $R^{0} \pi_{*}$ of (4.4) should be isomorphic to

$$
0 \rightarrow T_{\widetilde{S} / \widetilde{\mathcal{M}}} \rightarrow T_{\widetilde{S}}(\log W) \rightarrow \widetilde{f}^{*} T_{\widetilde{\mathcal{M}}}(B) \rightarrow 0,
$$

implying the assertion.

q.e.d.

\section{Remark 4.7.}

i) $R^{1} \pi_{*}\left(\mathcal{G}_{E}\right) \cong R^{0} \pi_{*}\left(\mathcal{E} n d(\mathbb{E})^{-1} \rightarrow \mathcal{A}_{\mathbb{E}, \pi}(\log D)\right) \cong \mathcal{D} \underset{\widetilde{S} / \widetilde{\mathcal{M}}}{\leq 1}\left(\lambda_{\mathbb{E}}\right)$ holds, cf. Theorem 3.7 and Remark 3.8.

ii) For the family $\widetilde{\mathcal{C}} \rightarrow \widetilde{\mathcal{M}}$ there is a Kodaira-Spencer map (cf. [20], $3.1-2)$

$$
\rho_{b}: T_{\widetilde{\mathcal{M}}, b} \rightarrow \operatorname{Ext}_{\mathcal{O}_{\widetilde{\mathcal{C}}_{b}}}^{1}\left(\Omega_{\widetilde{\mathcal{C}}_{b}}, \mathcal{O}_{\widetilde{\mathcal{C}_{b}}}\right), b \in \widetilde{\mathcal{M}}
$$

The family $\widetilde{\mathcal{C}} \rightarrow \widetilde{\mathcal{M}}$ is a local universal family if $\rho_{b}$ is an isomorphism at each $b \in \widetilde{\mathcal{M}}$. If $\widetilde{\mathcal{C}} \rightarrow \widetilde{\mathcal{M}}$ is a local universal family (cf. [20], Theorem 3.1 .5 for the existence of such a family), then KS (4.2) is an isomorphism (cf. [20], Theorem 3.2.6).

Combining the above with the second half of Section 3, we are now ready to generalize Theorem 3.7 in the context of log geometry.

Theorem 4.8. Suppose KS in (4.2) is an isomorphism (cf. Remark $4.7 \mathrm{ii})$. Then, over any affine open set $U_{i}$ of $\widetilde{\mathcal{M}}$, there is an isomorphism

$$
\phi_{i}: R^{1} \pi_{*} S\left(\mathcal{G}_{E}\right) \cong \mathcal{D} \underset{\widetilde{S}}{\leq 1}\left(\lambda_{\mathbb{E}}\right)(\log W) .
$$

Proof. Note the above assumption for KS is, via ii) of Proposition 4.5, in correspondence to $\mathrm{KS}_{S}$ in Theorem 3.7. It follows that all key ingredients in proof of Theorem 3.7 admit corresponding counterparts for log geometry, such as Proposition 4.3 and Proposition 4.5. Thus the generalization of Theorem 3.7 in $\log$ context is immediate. q.e.d.

To complete this paper, we prove the coherence of $f_{T *} \Theta^{k}$ (for definitions of $f_{T}$ and $f_{Z}$, see Notation 2.10), for which our proof needs a result on the density of locally free sheaves. 
Proposition 4.9. The fibre of $f_{Z}: Z \rightarrow \widetilde{\mathcal{M}}$ at any point of $B=$ $\widetilde{\mathcal{M}} \backslash \mathcal{M}$ has a dense open set of locally free sheaves.

Proof. Let $X_{0}$ be a fibre of $\mathcal{C} \rightarrow \widetilde{\mathcal{M}}$ at $0 \in B$. If $X_{0}$ is reducible, the lemma is known (see Theorem 1.6 and Lemma 2.2 of [18]). Thus we assume that $X_{0}$ is irreducible. Let $\mathcal{U}_{0}$ be the moduli space of semistable sheaves of rank $r$ and degree $d$ (without fixed determinant) on $X_{0}$. We need to show that $Z_{0}:=f_{Z}^{-1}(0)(\subset \mathcal{U})$ contains a dense set of locally free sheaves with the fixed determinant $\mathcal{N}_{0}$. Let $J\left(X_{0}\right)$ be the Jacobian of $X_{0}$, which consists of line bundles of degree 0 (thus noncompact for the singular curve $X_{0}$ ). Then we have a morphism

$$
\phi_{0}: Z_{0} \times J\left(X_{0}\right) \rightarrow \mathcal{U}_{0}, \quad \text { where } \phi_{0}(E, L)=E \otimes L .
$$

Now we prove that $\phi_{0}$ has fibre dimension at most 1 , namely, for any $\left[F_{0}\right] \in \mathcal{U}_{0}$, the fibre

$$
\phi_{0}^{-1}\left(\left[F_{0}\right]\right)=\left\{(F, L) \mid F \otimes L=F_{0}\right\} \subset Z_{0} \times J\left(X_{0}\right)
$$

has at most dimension 1 (for simplicity, we assume that $X_{0}$ has only one node $x_{0}$ ). One can check that for any $[F] \in Z_{0}$ it satisfies

$$
\frac{\wedge^{r} F}{\text { torsion }} \subset \mathcal{N}_{0} \text { and } m_{x_{0}}^{r(F)} \mathcal{N}_{0} \subset \frac{\wedge^{r} F}{\text { torsion }}
$$

where $m_{x_{0}}$ is the ideal sheaf of the node $x_{0} \in X_{0}$ and $r(F)=d-$ $\operatorname{deg}\left(\frac{\wedge^{r} F}{\text { torsion }}\right)$. Let $\rho: \widetilde{X_{0}} \rightarrow X_{0}$ be the normalization and $\rho^{-1}\left(x_{0}\right)=$ $\left\{x_{1}, x_{2}\right\}$. Then the above condition implies that

$$
\frac{\rho^{*}\left(\wedge^{r} F\right)}{\text { torsion }}=\rho^{*} \mathcal{N}_{0}\left(-n_{1} x_{1}-n_{2} x_{2}\right), \quad n_{1}+n_{2} \leq 2 r(F), n_{1} \geq 0, n_{2} \geq 0,
$$

where $r(F)=d-\operatorname{deg}\left(\frac{\wedge^{r} F_{0}}{\text { torsion }}\right)=r\left(F_{0}\right)$ since $F \otimes L=F_{0}$ and thus

$$
\frac{\wedge^{r} F_{0}}{\text { torsion }}=\frac{\wedge^{r} F}{\text { torsion }} \otimes L^{r} \text {. }
$$

Thus, for any $(F, L) \in \phi_{0}^{-1}\left(F_{0}\right), L$ has to satisfy

$$
\left(\rho^{*} L\right)^{r}=\left(\rho^{*} \mathcal{N}_{0}\right)^{-1}\left(n_{1} x_{1}+n_{2} x_{2}\right) \otimes \frac{\rho^{*} \wedge^{r} F_{0}}{\text { torsion }}
$$

which is a finite set since there are only finitely many choices of $\left(n_{1}, n_{2}\right)$. The pullback map $\rho^{*}: J\left(X_{0}\right) \rightarrow J\left(\widetilde{X_{0}}\right)$ has 1-dimensional kernel. Thus $\operatorname{dim}\left(\phi_{0}^{-1}\left(\left[F_{0}\right]\right)\right) \leq 1$. We shall now prove the density of locally free sheaves. 
Let $Z_{0}^{i}$ be an irreducible component of $Z_{0}$, then

$$
\operatorname{dim}\left(Z_{0}^{i} \times J\left(X_{0}\right)\right) \geq \operatorname{dim}\left(Z_{\eta} \times J\left(X_{0}\right)\right)=\operatorname{dim}\left(\mathcal{U}_{0}\right) .
$$

If $Z_{0}^{i}$ contains no locally free sheaf, then $\phi\left(Z_{0}^{i} \times J\left(X_{0}\right)\right)$ falls into the subvariety $\mathcal{U}_{0}^{n} \subset \mathcal{U}_{0}$ of nonlocally free sheaves. $\mathcal{U}_{0}^{n}$ has a dense open set $\mathcal{U}_{0}^{n}(1)$ consisting of torsion-free sheaves $F$ of the following type, said to be type 1. Namely:

$$
F \otimes \hat{\mathcal{O}}_{X_{0}, x_{0}}=\hat{\mathcal{O}}_{X_{0}, x_{0}}^{(r-1)} \oplus \hat{m}_{x_{0}}
$$

If $\phi((F, L))=F_{0} \in \mathcal{U}_{0}^{n}(1)$, then $F$ is also of type 1 (tensoring a line bundle does not change its type). By Remark 8.1 of [15],

$$
L_{0}=\frac{\wedge^{r} F}{\text { torsion }}
$$

is a torsion-free (but nonlocally free) sheaf of degree $d$. But $L_{0} \subset \mathcal{N}_{0}$, thus $L_{0}=\mathcal{N}_{0}$ since they have the same degree, a contradiction with that $L_{0}$ is not locally free. Thus $\phi\left(Z_{0}^{i} \times J\left(X_{0}\right)\right)$ falls into a subvariety of codimension at least two, which contradicts the dimension of fibres and (4.6).

q.e.d.

\section{Theorem 4.10.}

i) $f_{T *} \Theta^{k}$ is coherent.

ii) $f_{T *} \Theta^{k}=\widetilde{f}_{*} \Theta^{k}$ if either $g \geq 3$ or $r \geq 3$.

Proof. Let $\iota: \widetilde{Z} \rightarrow Z$ be the normalisation of $Z$ and $\widetilde{\Theta}=\iota^{*}(\Theta)$. Write $f_{\widetilde{Z}}: \widetilde{Z} \rightarrow \widetilde{\mathcal{M}}$ and $f_{\iota^{-1}(T)}: \iota^{-1}(T) \rightarrow \widetilde{\mathcal{M}}$. Then $\iota^{-1}(T) \cong T$ since $T$ is normal, and $\mathcal{F}:=f_{T *} \Theta^{k} \cong f_{\iota^{-1}}(T) *\left(\widetilde{\Theta}^{k}\right)$. On the other hand, since each fibre of $f_{Z}: Z \rightarrow \widetilde{\mathcal{M}}$ contains a dense open set of locally free sheaves, we have $\operatorname{codim}(Z \backslash T) \geq 2$. Thus $\widetilde{Z} \backslash \iota^{-1}(T)=\iota^{-1}(Z \backslash T)$ has codimension at least 2 since $\iota: \widetilde{Z} \rightarrow Z$ is a finite map. By Hartogs type extension theorem,

$$
\mathcal{F} \cong f_{\iota^{-1}(T) *}\left(\widetilde{\Theta}^{k}\right) \cong f_{\widetilde{Z} *}\left(\widetilde{\Theta}^{k}\right),
$$

which is coherent, hence i). The claim ii) that $f_{T *} \Theta^{k}=\widetilde{f}_{*} \Theta^{k}$ follows also from the Hartogs type theorem because $T$ is normal and $T \backslash \widetilde{S}$ is of codimension at least 2 when $g>2$ or $r>2$ (cf. [10]).

q.e.d.

Finally, we prove the following lemma, though not strictly needed for this paper, which gives the relationships of $S\left(\mathcal{G}_{E}\right), \mathcal{G}_{E^{*}}$ and $\mathcal{A}_{E^{*} / S}$. We remark that the morphism $\widetilde{\text { res }}$ in Proposition 3.13 can be induced by pairings in this lemma. 
Lemma 4.11. There are canonical isomorphisms:

i) $S\left(\mathcal{G}_{E}\right) / \omega_{X / S} \cong\left(\mathcal{G}_{E^{*}}\right)^{*}$,

ii) $\left(\mathcal{G}_{E}\right)^{*} \cong \mathcal{A}_{E^{*} / S}^{0}$ where $\mathcal{A}_{E^{*} / S}^{0}=\mathcal{A}_{E^{*} / S} / \mathcal{O}_{X}$.

\section{Proof.}

i) One constructs a nondegenerate pairing

$$
\mathcal{G}_{E^{*}} \times S\left(\mathcal{G}_{E}\right) / \omega_{X / S} \rightarrow \mathcal{O}_{X}
$$

using local description (3.7) (also cf. [2]). We define

$$
\left\langle s_{1}, s_{2}\right\rangle:=\chi \nu+\frac{1}{2} \sum_{a}\left(\nu_{a}+\mu_{a}\right) \operatorname{Trace}\left(J_{a} \cdot{ }^{t} B\right)
$$

for $s_{1}=(0, B, \nu)$ and

$$
s_{2}=\left(\begin{array}{c}
\chi \sum_{a}\left(0, J_{a}, 0\right) \otimes\left(0, J_{a}, 0\right)+\sum_{a} \mu_{a}\left(0, J_{a}, 0\right) \otimes(0,0,1) \\
+\sum_{a} \nu_{a}(0,0,1) \otimes\left(0, J_{a}, 0\right)+w(0,0,1) \otimes(0,0,1)
\end{array}\right) \otimes \partial_{t} .
$$

One sees that $\omega_{X / S}$ is contained in the kernel of the pairing (4.7). The pairing is obviously invariant under coordinates change $y(t)$. If the trivialization of $E$ is changed by a gauge $g$, then $E^{*}$ is changed by a gauge $\left({ }^{t} g\right)^{-1}$. Thus the verification of (4.7) being $g$-invariant is easily reduced to an identity

$$
\sum_{a} \operatorname{Tr}\left(-J_{a} g^{-1} g^{\prime}\right) \cdot \operatorname{Tr}\left(J_{a} \cdot{ }^{t} B\right)=\operatorname{Tr}\left(B\left({ }^{t} g\right)\left(\left({ }^{t} g\right)^{-1}\right)^{\prime}\right) .
$$

The left-hand side of (4.8) equals $\operatorname{Tr}\left(-g^{-1} g^{\prime} \cdot{ }^{t} B\right)$ ( $B$ being traceless). The right-hand side of (4.8), after transposition, is

$$
\operatorname{Tr}\left(\left(g^{-1}\right)^{\prime} g \cdot{ }^{t} B\right)=\operatorname{Tr}\left(\left(-g^{-1} g^{\prime} g^{-1}\right) g \cdot{ }^{t} B\right) .
$$

ii) Define a nondegenerate pairing

$$
\mathcal{G}_{E} \times \mathcal{A}_{E^{*} / S}^{0} \rightarrow \mathcal{O}_{X}
$$


by $\left\langle\left(0, B_{1}, \nu\right),\left(\chi, B_{2}\right)\right\rangle=\nu \chi+\operatorname{Trace}\left(B_{1} \cdot{ }^{t} B_{2}\right)$. We check that it is independent of choices of coordinates and gauges.

$$
\begin{aligned}
\left(0, B_{1}, \nu\right):= & s_{1} \stackrel{g}{\longrightarrow}\left(0, g B_{1} g^{-1}, \operatorname{Tr}\left(-B_{1} g^{-1} g^{\prime}\right)+\nu\right) ; \\
\left(\chi, B_{2}\right):= & s_{2} \stackrel{{ }^{t} g^{-1}}{\longrightarrow}\left(\chi,-\chi\left(\left({ }^{t} g^{-1}\right)^{\prime} \cdot{ }^{t} g\right)+\left({ }^{t} g^{-1}\right) B_{2} \cdot{ }^{t} g\right) ; \\
& s_{1} \stackrel{y(t)}{\longrightarrow}\left(0, B_{1}, \nu y^{\prime}\right) ; \\
& s_{2} \stackrel{y(t)}{\longrightarrow}\left(\chi y^{\prime-1}, B_{2}\right) .
\end{aligned}
$$

The $y(t)$-change is obvious. For $g$-change, we have

$$
\begin{aligned}
\chi \nu+\operatorname{Tr}\left(B_{1} \cdot{ }^{t} B_{2}\right) \longrightarrow \chi \nu & +\chi \operatorname{Tr}\left(-B_{1} g^{-1} g^{\prime}\right) \\
& +\operatorname{Tr}\left(g B_{1} g^{-1} \cdot{ }^{t}\left(-\chi\left(\left({ }^{t} g^{-1}\right)^{\prime} \cdot{ }^{t} g\right)\right)\right. \\
& +\operatorname{Tr}\left(g B_{1} g^{-1} \cdot{ }^{t}\left({ }^{t} g^{-1} B_{2} \cdot{ }^{t} g\right)\right) .
\end{aligned}
$$

The first (resp. second) term in the last line equals

$$
\begin{aligned}
-\chi \operatorname{Tr}\left(g B_{1} g^{-1} \cdot g\left(g^{-1}\right)^{\prime}\right) & =-\chi \operatorname{Tr}\left(g B_{1} g^{-1} \cdot g\left(-g^{-1} g^{\prime} g^{-1}\right)\right) \\
& =\chi \operatorname{Tr}\left(g\left(B_{1} g^{-1} g^{\prime}\right) g^{-1}\right) \\
& =\chi \operatorname{Tr}\left(B_{1} g^{-1} g^{\prime}\right) \\
\text { (resp. } \operatorname{Tr}\left(g B_{1} \cdot{ }^{t} B_{2} g^{-1}\right) & \left.=\operatorname{Tr}\left(B_{1} \cdot{ }^{t} B_{2}\right)\right) .
\end{aligned}
$$

It follows from (4.11) and (4.12) that the pairing $\chi \nu+\operatorname{Tr}\left(B_{1}{ }^{t} B_{2}\right)$ in (4.9) is globally defined.

q.e.d.

\section{References}

[1] A. Beilinson \& D. Kazhdan, Flat projective connections, unpublished manuscript.

[2] A. Beilinson \& V. Schechtman, Determinant bundles and Virasoro algebras, Commun. Math. Phys. 118 (1988) 651-701, MR 0962493 (90m:32048), Zbl 0665.17010.

[3] J.L. Brylinski \& D. McLaughlin, Holomorphic quantization and unitary representations of the Teichmüller group, in 'Lie Theory and Geometry' in honor of Bertram Kostant, J.L. Brylinski et al., Progress in Mathematics, 123, Birkhäuser Boston, 1994, MR 1327530 (97d:32041), Zbl 0846.58030.

[4] H. Esnault \& I-H. Tsai, Determinant bundle in a family of curves, after A. Beilinson and V. Schechtman, Commun. Math. Phys. 211 (2000) 359-363, MR 1754519 (2001c:14024), Zbl 0956.58016. 
[5] G. Faltings, Stable G-bundles and projective connections, J. of Alg. Geom. 2 (1993) 507-568, MR 1211997 (94i:14015), Zbl 0790.14019.

[6] G. Faltings, A proof for the Verlinde formula, J. of Alg. Geom. 3 (1994) 347-374, MR 1257326 (95j:14013), Zbl 0809.14009.

[7] G. Faltings, Moduli-stacks for bundles on semistable curves, Math. Ann. 304 (1996) 489-515, MR 1375622 (97d:14016), Zbl 0847.14018.

[8] B. van Geemen \& J. de Jong, On Hitchin's connection, J. Amer. Math. Soc. 11(1) (1998) 189-228, MR 1469656 (99c:14015), Zbl 0920.32017.

[9] V. Ginzburg, Resolution of diagonals and moduli spaces, in 'Moduli space of curves', R. Dijkgraaf et al., Progress in Mathemtics, 129, Birkhäuser Boston, 1995, MR 1363059 (96j:14003), Zbl 0841.14006.

[10] N. Hitchin, Flat connections and geometric quantization, Commun. Math. Phys. 131 (1990) 347-380, MR 1065677 (91g:32022), Zbl 0718.53021.

[11] D. Huybrechts \& M. Lehn, The geometry of moduli spaces of sheaves, Aspects of Mathematics, 31, A publication of the Max-Planck-Institut für Mathematik, Bonn, Vieweg, 1997, MR 1450870 (98g:14012), Zbl 0872.14002.

[12] N. Katz, Algebraic solutions of differential equations, p-curvature and the Hodge filtration, Invent. Math. 87 (1972) 1-118, MR 0337959 (49 \#2728), Zbl 0278.14004.

[13] Y. Laszlo, Hitchin's and WZW connections are the same, J. Differential Geom. 49 (1998) 547-576, MR 1669720 (2000e:14012), Zbl 0987.14027.

[14] E. Looijenga, Smooth Deligne-Mumford compactifications by means of Prym level structures, J. of Alg. Geom. 3 (1992) 283-293, MR 1257324 (94m:14029), Zbl 0814.14030.

[15] D.S. Nagaraj \& C.S. Seshadri, Degenerations of the moduli spaces of vector bundles on curves I, Proc. Indian Acad. Sci. (Math. Sci.) 107(2) (1997) 101137, MR 1455315 (98f:14008), Zbl 0922.14023.

[16] Z. Ran, Jacobi cohomology, local geometry of moduli spaces, and Hitchin's connection, math.AG/0108101.

[17] C.S. Seshadri, Degenerations of the moduli spaces of vector bundles on curves, ICTP Lecture Notes, 1, Moduli spaces in Algebraic Geometry, 1999, MR 1795864 (2001i:14044), Zbl 0986.14018.

[18] X.-T. Sun, Degeneration of $S L(n)$-bundles on a reducible curve, Proceedings of the Symposium on Algebraic Geometry in East Asia (Kyoto, August 3-10, 2001), 229-243, World Scientific, 2003, MR 2030457.

[19] Y.-L. Tong \& I-H. Tsai, Curvature of determinant bundles for degenerate families, Commun. Math. Phys. 171 (1995) 589-606, MR 1346173 (96j:58177), Zbl 0859.32016. 
[20] A. Tsuchiya, K. Ueno \& Y. Yamada, Conformal field theory on universal family of stable curves with gauge symmetries, Adv. Stud. in Pure Math. 19 (1989) 459-566, MR 1048605 (92a:81191), Zbl 0696.17010.

INSTITUTE OF MATHEMATICS AMSS, Chinese ACADEmy of ScIEnces China

E-mail address: xsun@mail2.math.ac.cn and Department of Mathematics The University of Hong Kong POKFulam RoAd, Hong Kong

E-mail address: xsun@maths.hku.hk

Department of Mathematics National Taiwan University, Taipei TAIWAN

E-mail address: ihtsai@math.ntu.edu.tw 